\title{
Consideraciones sobre la sistemática de las familias y los géneros de plantas vasculares endémicos de Chile
}

\section{Systematic considerations of Chilean endemic vascular plant families and genera}

\author{
Rafael Urbina-Casanova ${ }^{1 *}$, Patricio Saldivia ${ }^{2} \&$ Rosa A. Scherson ${ }^{1}$ \\ ${ }^{1}$ Laboratorio de Sistemática y Evolución de Plantas, Departamento de Silvicultura y Conservación de la Naturaleza, Universidad \\ de Chile. Av. Santa Rosa 11.315, La Pintana, Santiago, Chile. \\ ${ }^{2}$ Biota, Gestión y Consultorías Ambientales Ltda. Av. Miguel Claro 1.224, Providencia, Santiago, Chile. \\ *rafaelurbinac@gmail.com
}

\begin{abstract}
RESUMEN
El endemismo es uno de los principales aspectos que trata la biogeografía histórica y es uno de los criterios más importantes para establecer las prioridades de conservación de las especies. En el mundo, más del $90 \%$ de las plantas que se encuentra en alguna categoría de amenaza son endémicas de un sólo país. En Chile, un $45 \%$ de las especies de plantas vasculares son endémicas. Actualmente este número incluye 83 géneros y 4 familias endémicas del país; éstos son valores elevados en comparación con el resto de Latinoamérica. Sin embargo, la alta tasa de cambios producidos por los estudios de sistemática molecular en la taxonomía ha generado modificaciones en estos números. Este trabajo pretende discutir dichas modificaciones y así contribuir a la correcta delimitación de estos géneros endémicos. Utilizando bases de datos y bibliografía actualizadas, se llevó a cabo una revisión exhaustiva sobre estos géneros. Se sustrajeron de la lista aquellos géneros con registros fuera del país y aquellos que cuentan con evidencia suficiente para cambiar su estatus taxonómico. Se entrega una revisión crítica de la bibliografía sistemática respecto de los géneros en cuestión, discutiendo principalmente aquellos que a la luz de la literatura presentaron ambigüedades en su clasificación.
\end{abstract}

Palabras clave: Endemismo, Sistemática molecular, Filogenia molecular, Conservación biológica.

\begin{abstract}
Endemism is one of the main considerations in historical biogeography and one of the most important criteria when establishing conservation priorities. Worldwide, more than $90 \%$ of the plants listed in conservation categories are endemic to one country. In Chile, $45 \%$ of the flora is endemic, currently considering 83 endemic genera and 4 endemic families, elevated numbers compared to the rest of Latinamerica. However, the high rate of changes in taxonomy, based on studies of molecular systematics has produced modifications in this list. This study aims at discussing such modifications and in this way, contribute to the correct delimitation of our endemic genera. Using updated databases and bibliographic sources, an exhaustive revision of these genera was carried out. Genera with registries outside of the country, as well as those that had enough evidence to change their taxonomic status were subtracted from the list. A critical revision of the systematic bibliography available is provided, emphasizing the discussion on those genera with ambiguous classification status.
\end{abstract}

KeYwords: Endemism, Molecular systematics, Molecular phylogeny, Biological conservation.

\section{INTRODUCCIÓN}

El estudio de los endemismos es uno de los objetivos principales de la biogeografía histórica y es uno de los criterios más importantes para establecer las prioridades de conservación(Linder2001).Esteconceptoestáimplícitamente relacionado con la idea de taxa irreemplazables de Margules \& Pressey (2000) y, en consecuencia, con la importancia tanto de los taxa en sí mismos, como de las áreas geográficas que los contienen. Un territorio se considera como un área de endemismo cuando alberga al menos dos taxa endémicos no relacionados directamente entre sí (Harold \& Mooi 1994). Si se observa, por ejemplo, la frecuencia de endemismo entre las especies clasificadas en alguna categoría de amenaza por la UICN, se tiene que, a escala global, más del $90 \%$ son endémicas de un sólo país (Pitman \& Jorgensen 2002). Esto es importante si se considera que, en general, los países son soberanos sobre su territorio y recursos naturales, y por esto la conservación de estas especies está supeditada a las políticas de cada uno de ellos (Pitman \& Jorgensen 2002). 
Según Moreira-Muñoz (2011) la riqueza de flora vascular autóctona de Chile alcanza a unas 4.295 especies, que se agrupan en 837 géneros. De ellas, actualmente un $45 \%$ se consideran endémicas. Si se considera lo estimado para las regiones de Atacama y Coquimbo, el endemismo llega a un 54,3\% (Letelier et al. 2008) y a un 53,5\% (Squeo et al. 2001) respectivamente. Por otro lado, en el caso insular, las Islas Desventuradas y el Archipiélago de Juan Fernández presentan los niveles más altos de endemismo vegetal en Chile con un 86,9 y $64,3 \%$ de sus especies autóctonas, respectivamente (Marticorena 1990, Danton et al. 2006). El archipiélago de Juan Fernández muestra, además, la mayor densidad insular de especies endémicas por unidad de superficie a nivel mundial (Smith-Ramírez et al. 2013).

Según Moreira-Muñoz (2011) en Chile existen 83 géneros y 4 familias endémicas, situación excepcional en Latinoamérica. Argentina, por ejemplo, tiene una familia y cuatro géneros endémicos (Zuloaga et al. 1999); Perú, con una flora de más de 18.000 especies de plantas vasculares, tiene 51 géneros y ninguna familia endémica (Brako \& Zarucchi 1993, Ulloa et al. 2004); Ecuador, con una flora agrupada en aproximadamente 2.110 géneros, sólo presenta 23 endémicos (Jorgensen \& León-Yáñez 1999). En Chile la distribución geográfica de estos géneros se concentra en la zona central (Moreira-Muñoz 2011, Scherson et al. 2014), en el hotspot de biodiversidad (Myers et al. 2000, Mittermeier et al. 2004), que contiene a los ecosistemas más amenazados y menos protegidos del país (Luebert \& Becerra 1998, Pliscoff \& Fuentes-Castillo 2011).

En escenarios de cambio global, los géneros y familias endémicos de Chile son particularmente importantes, ya que la mayor parte de ellos son monoespecíficos o presentan una baja riqueza (Moreira-Muñoz 2011). Esto es relevante ya que estudios recientes han demostrado que las probabilidades de extinción en angiospermas aumentan en las familias con pocas especies (Vamosi \& Wilson 2008), lo que implica que si una o las pocas especies del taxon desaparecen, se extinguiría con ellas la historia evolutiva de un grupo completo. En este sentido, la incorrecta delimitación sistemática de las familias y de los géneros endémicos puede ser un obstáculo para su conservación (Duarte et al. 2014).

El estudio de los géneros endémicos de Chile es importante no sólo desde el punto de vista de su conservación, sino porque también constituyen una herramienta para contestar preguntas de interés biológico, especialmente de tipo biogeográfico. Sin embargo, la delimitación taxonómica y posicionamiento sistemático de estos grupos no es ni ha sido un proceso sencillo. En las últimas décadas y en virtud del amplio desarrollo de la genética molecular, se ha generado una enorme cantidad de estudios filogenéticos tendientes al reconocimiento sistemático y taxonómico de grupos monofiléticos para así obtener una representación más fidedigna de la historia evolutiva de los organismos. Este proceso de reorganización sistemática y taxonómica se encuentra en pleno desarrollo, produciéndose modificaciones constantes a las clasificaciones tradicionales, las que en muchos casos no están exentas de controversia. Esta inestabilidad taxonómica se ha ido resolviendo paulatinamente en los niveles taxonómicos superiores, como Órdenes y Familias, y sigue avanzando del mismo modo hacia los niveles inferiores, concentrándose aquí la mayoría de las modificaciones en curso. El presente trabajo pretende ser un aporte a la construcción de una lista de los géneros endémicos de Chile, y como una fuente de referencia para el estudio general o particular de las familias y los géneros en discusión.

\section{MATERIALES Y MÉTODOS}

Basados en la lista de géneros endémicos de Chile recopilada por Moreira-Muñoz (2011), se hizo una comparación con el "Catálogo de las Plantas Vasculares del Cono Sur" (Zuloaga et al. 2008), disponible y actualizado en la página web del Instituto de Botánica Darwinion de Argentina ${ }^{1}$ (revisado hasta el 2 de junio de 2014), al que nos referiremos en adelante como "el Catálogo" sin referencia bibliográfica, dado que su uso será muy extensivo en el trabajo. Ambas referencias corresponden a las recopilaciones más actuales y ampliamente utilizadas. Se presenta además una amplia revisión de la literatura sistemática disponible, con énfasis en aquellos taxa que presentan controversias en su clasificación. Para ello consideramos principalmente los análisis filogenéticos utilizados para proponer cambios en la nomenclatura, enfocándonos en los soportes estadísticos que sustentan dichos cambios. Se incluyó además literatura que diera cuenta de nuevos géneros no reconocidos en Moreira-Muñoz (2011) y/o el Catálogo.

Por otro lado, la distribución geográfica de los géneros se corroboró mediante los datos disponibles en $\mathrm{GBIF}^{2}$ (Global Biodiversity Information Facility), los cuales se basan principalmente en la información digitalizada de numerosos herbarios a nivel mundial (Edwards et al. 2000). En casos puntuales se revisaron especímenes digitales y se consultaron especialistas para corroborar la identificación de los materiales que se encontraban fuera de Chile, los cuales son citados en la discusión de cada grupo.

Se propone una actualización de la lista de géneros y familias endémicos de Chile a la luz de los antecedentes encontrados, discutiendo principalmente aquellos que presentaron ambigüedades en su clasificación. No obstante, aún deben resolverse algunas dudas que necesitan más y mejores análisis, las cuales seguramente irán despejándose con el trabajo de los especialistas en cada grupo.

Finalmente, utilizando la nueva lista, se realizó una descripción del estado de amenaza de los géneros endémicos, en base a las categorías de conservación para cada una

\footnotetext{
1 http://www2.darwin.edu.ar/Proyectos/FloraArgentina/FA.asp.

2 http://www.gbif.org/
} 
de las especies, según Benoit (1989), el reglamento de clasificación de especies (D.S. 75/2005 del MINSEGPRES mod. D.S. 29/2012 del MMA) hasta el décimo proceso y la UICN (2013).

\section{RESULTADOS}

De las cuatro familias endémicas tradicionalmente reportadas para Chile: Francoaceae, Gomortegaceae, Lactoridaceae y Thyrsopteridaceae, el estatus taxonómico de Francoaceae, Lactoridaceae y Thyrsopteridaceae ha sido objeto de controversia (ver discusión para detalles).

De los 83 géneros endémicos de Chile reportados por Moreira-Muñoz (2011), 65 exclusivamente continentales, 16 insulares y 2 de distribución compartida, en el Catálogo se reconocen 78, 61 exclusivamente continentales, 15 exclusivamente insulares y 2 de distribución compartida. Los géneros Epipetrum, Gymnachne, Miqueliopuntia, Phrodus y Robinsonia no son reconocidos por dicha publicación debido a que distintos autores han propuesto su inclusión en otros géneros (ver discusión más adelante). Del Catálogo se desprenden, además, dos géneros (Lardizabala y Famatina) que no fueron considerados como endémicos por Moreira-Muñoz (2011); sin embargo, Famatina ha sido recientemente reportado como parafilético (García et al. 2014), lo que se discute en detalle más adelante. Adicionalmente, producto de la revisión bibliográfica se reportan Aimara, Ancrumia, Atacama, Gilliesia, Kieslingia, Nothomyrcia, Phycella y Rimacactus, como géneros endémicos no considerados ni en Moreira-Muñoz (2011) ni en el Catálogo, siendo Gilliesia y Phycella excepcionales por ser objeto de controversia. Producto de la misma revisión se restaron tres géneros, principalmente producto de sinonimias recientes y de registros fuera del país, los que corresponden a Famatina, Homalocarpus y Eulychnia. Se discuten en detalle 45 géneros, incluyendo las adiciones, las sustracciones y aquéllos cuyos antecedentes encontrados no son suficientes para dilucidar su situación con certeza. Proponemos que Chile cuenta actualmente con 90 géneros endémicos, 70 exclusivamente continentales, 17 exclusivamente insulares y 3 de distribución compartida, cifra que considera como endémicos los géneros de estatus dudoso o controversial mencionados anteriormente, cuya situación no puede ser resuelta sin análisis adicionales.

Respecto del estado de conservación, se observó que un $37 \%$ de los géneros endémicos continentales tienen al menos una especie en alguna categoría de amenaza, mientras que en las islas, un $59 \%$ de los géneros tienen al menos una especie amenazada. Del mismo modo, se observó que un 49\% de las especies de Chile continental amenazadas se encuentran en las categorias En Peligro, En Peligro Crítico o Extinta, mientras que en el caso de las islas un $97 \%$ de las especies amenazadas se encuentra en alguna de las tres categorías mencionadas. Los géneros continentales con más especies clasificadas en algún grado de amenaza son Copiapoa (23 spp.) y Neoporteria (6 spp.), mientras que en las islas son Dendroseris (11 spp.) y Robinsonia (8 spp.). Finalmente, señalar que las islas presentan un mayor número de extinciones (Robinsonia berteroi, Robinsonia macrocephala y Podophorus bromioides) en comparación a Chile continental (Ivania cremnophila).

\section{DISCUSIÓN}

Con respecto a las cuatro familias endémicas reconocidas para Chile, Francoaceae (incluyendo Greyiaceae) ha sido propuesta como parte de Melianthaceae (Savolainen et al. 2000, APG III 2009), sin embargo, Applequist (2012) señala que el Comité para la Nomenclatura de Plantas Vasculares no llegó a acuerdo respecto de esta solicitud, conservando ambos nombres como válidos, con Francoaceae como nombre prioritario. Palazzesi et al. (2012) presentan una filogenia bien resuelta que clarifica la mayoría de las relaciones entre las Geraniales, exceptuando la relación entre Melianthaceae, Francoaceae (incluyendo Greyiaceae) y Vivianiaceae. Por lo tanto, con la información actualmente disponible, no es posible poner a prueba la hipótesis de Melianthaceae s.l., la cual podría o no ser parafilética según como se resuelvan dichas relaciones. No obstante, tanto Palazzesi et al. (2012) como otros estudios anteriores (Chase et al. 1993, Morgan \& Soltis 1993, Price \& Palmer 1993, Soltis \& Soltis 1997) señalan claramente que Francoaceae es un clado hermano de Greyiaceae; por lo que los autores proponen agrupar a ambos clados en una única familia, extendiendo así el concepto de Francoaceae. Si se acepta el cambio propuesto, Francoaceae dejaría de ser una familia endémica de Chile, distribuyéndose también en Sudáfrica. No obstante, tanto fusionar como mantener ambas familias son alternativas sistemáticamente correctas. Por lo tanto, la búsqueda de sinapomorfias en dichos clados es necesaria para evaluar la coherencia de la propuesta a la luz de su historia evolutiva.

La familia Thyrsopteridaceae, monotípica y endémica de Juan Fernández, es considerada como parte de la familia Dicksoniaceae por el Catálogo, a pesar de que las últimas filogenias moleculares reconocen a ambas familias como clados independientes (Smith et al. 2006, Schuettpelz \& Pryer 2007, Kuo et al. 2011, Lehtonen 2011). Christenhusz et al. (2011) proponen una clasificación de los helechos sobre la base de los avances sistemáticos en el grupo, en la que la familia Thyrsopteridaceae sigue siendo reconocida. Sin embargo, Christenhusz \& Chase (2014) proponen una nueva clasificación para reunir a varias familias de helechos arbóreos, entre ellas Thyrsopteridaceae, en una única familia Cyatheaceae; para ello argumentan que dichos helechos son mínimamente divergentes genéticamente y que el tiempo de divergencia del clado es relativamente reciente y similar al 
de otras familias de angiospermas; señalan un carácter del annulus del esporangio como diagnóstico para el grupo completo, anteriormente utilizado para definir al orden Cyatheales, y además la necesidad de buscar caracteres diagnósticos al interior del grupo. De aceptarse esta propuesta taxonómica, la familia Thyrsopteridaceae quedaría en categoría de subfamilia, y la familia Cyatheaceae no sería endémica de Chile.

La familia Lactoridaceae, también monotípica y endémica de Juan Fernández, ha sido incluida en numerosos análisis filogenéticos, en los cuales se estableció su clara ubicación dentro de Piperales (Qiu et al. 1993, Chase et al. 1993, Soltis et al. 1997), y posteriormente una estrecha relación con Aristolochiaceae (Savolainen et al. 2000, Soltis et al. 2000, Qiu et al. 2000). Respecto a esta última asociación, no se ha logrado esclarecer con certeza la posición de Lactoris respecto a las dos subfamilias de Aristolochiaceae, la cual suele aparecer parafilética con Lactoris en su interior (Neinhuis et al. 2005, Naumann et al. 2013). Sin embargo, ni el sustento estadístico de esta topología, ni los análisis morfológicos que se han llevado a cabo, permiten adoptar una decisión coherente al respecto. La politomía de estos tres clados es, hasta el momento, la interpretación más correcta, no pudiéndose descartar con certeza una relación de clados hermanos entre ambas familias. Dado que Lactoridaceae es monotípica, es probable que su inclusión en Aristolochiaceae sea propuesta en favor de la estabilidad taxonómica del grupo, esté o no encestada en su interior. Sin embargo, esto debe hacerse a la luz de mayor evidencia molecular y de otras líneas de evidencia que permitan una interpretación concluyente.

En cuanto a los géneros endémicos y sus especies, se evidencian varios cambios taxonómicos con respecto del trabajo de Moreira-Muñoz (2011); esto no sorprende, dado el dinamismo de las clasificaciones producto del auge de las investigaciones en sistemática molecular. Sin embargo, se observaron tres situaciones recurrentes a discutir:

1) La modificación de jerarquías taxonómicas, como es el caso de la delimitación genérica en Vivianiaceae, donde se incluye en Viviania a los géneros Caesarea y Cissarobryon. Esto es un buen ejemplo de un cambio de jerarquía taxonómica basado en un análisis filogenético que también sustenta otras hipótesis, como por ejemplo que ambos géneros fueran el grupo hermano de Viviania (s.s.); la cual es otra interpretación correcta de la misma filogenia. Ejemplos similares son los mencionados anteriormente en relación con la delimitación del clado Francoaceae/Greyiaceae y los helechos arbóreos. Dado que las filogenias disponibles no presentan evidencia concluyente, estos cambios deberían sustentarse sobre información complementaria morfoanatómica y biogeográfica, entre otras.

2) Propuestas de cambios taxonómicos sobre la base de filogenias con bajo soporte estadístico. Un buen ejemplo de esto corresponde a la inclusión de Leontochir en Bomarea.
Si consideramos la evidencia molecular en sentido estricto, es cierto que ambos géneros forman un clado, sin embargo la topología del mismo no se encuentra resuelta, pudiendo darse dos soluciones que sustentan hipótesis taxonómicas alternativas: que Leontochir es un taxón basal, y en consecuencia podría ser considerado como el grupo hermano de Bomarea, o que se encuentre encestado en Bomarea. En este caso es necesario aumentar la resolución filogenética al mismo tiempo que se introducen otras líneas de evidencia en el debate. En este sentido, lo señalado por Aagesen \& Sanso (2003) respecto a que morfológicamente Bomarea no podría ser definido sin la inclusión de Leontochir es un argumento más fuerte que la evidencia molecular.

3) La inclusión de taxa no muestreados en las propuestas de cambios taxonómicos. La propuesta de cambios taxonómicos basados en estudios moleculares sin el muestreo de los taxa a modificar corresponde a una situación compleja, ya que son conjeturas válidas, pero faltas de evidencia. Un buen ejemplo de esto es la propuesta de inclusión de Epipetrum en Dioscorea; Caddick et al. (2002a, 2002b) hacen esta propuesta sin muestrear genéticamente a Epipetrum y se basan entonces en la afinidad morfológica del género con otros géneros que sí fueron considerados en la filogenia y quedan incluidos en Dioscorea. Otro ejemplo similar sería la inclusión de Thamnoseris en Sonchus, o Yunquea en Centaurodendron.

La adopción de cambios taxonómicos de manera periódica por parte de publicaciones disponibles online $\mathrm{y}$ que sirven de referencia nomenclatural, como el Catálogo de la Flora Vascular del Cono Sur, requiere de un cuidadoso criterio que juzgue la evidencia en uno u otro sentido ya que la aceptación de una u otra propuesta repercute en el tratamiento de los taxa por parte de investigadores que no son especialistas en los grupos y recurren a estas referencias en busca de una guía al respecto.

En el presente trabajo se ha propuesto exponer distintos escenarios, tomando decisiones basadas en la evidencia cuando ésta estaba disponible y era suficiente, principalmente en lo que concierne a la sistemática molecular. En la mayoría de los casos es necesario aumentar la resolución filogenética para sacar conclusiones definitivas o discutir distintas hipótesis sistemáticas al alero de otras líneas de evidencia. Por el momento, se detallan las propuestas y los motivos de la incertidumbre, ya que la solución de dichos problemas excede los objetivos de esta revisión.

A continuación se entrega una lista comentada de las adiciones, las sustracciones y aquellos géneros para los que se dispone de información filogenética actualizada o de algún otro antecedente que sea importante para su discusión. Se omiten de la discusión aquellos géneros que no han sido objeto de controversia y que han mantenido su estatus. La lista completa de los géneros endémicos y la bibliografía revisada se encuentra en las Tablas I, II y III. 


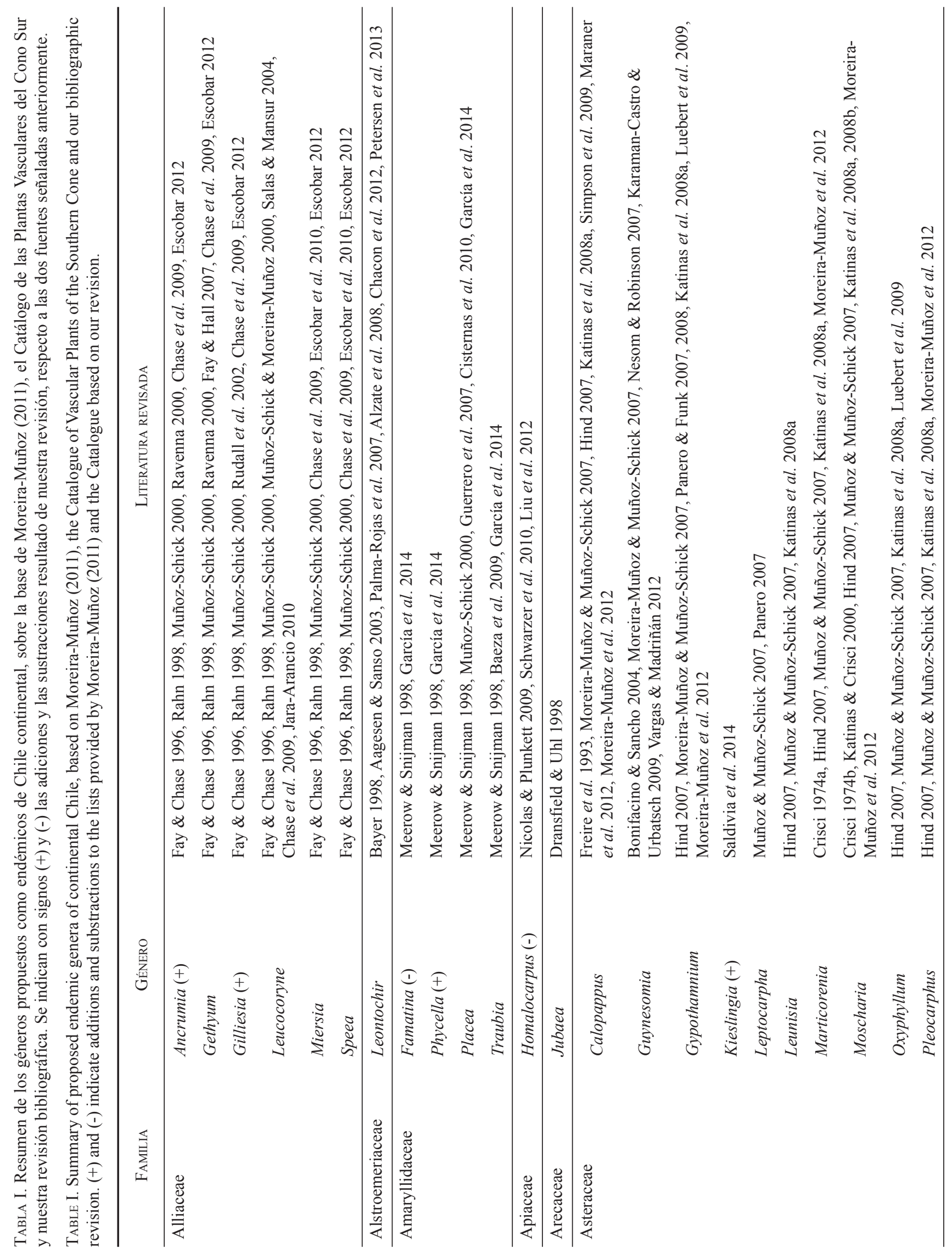




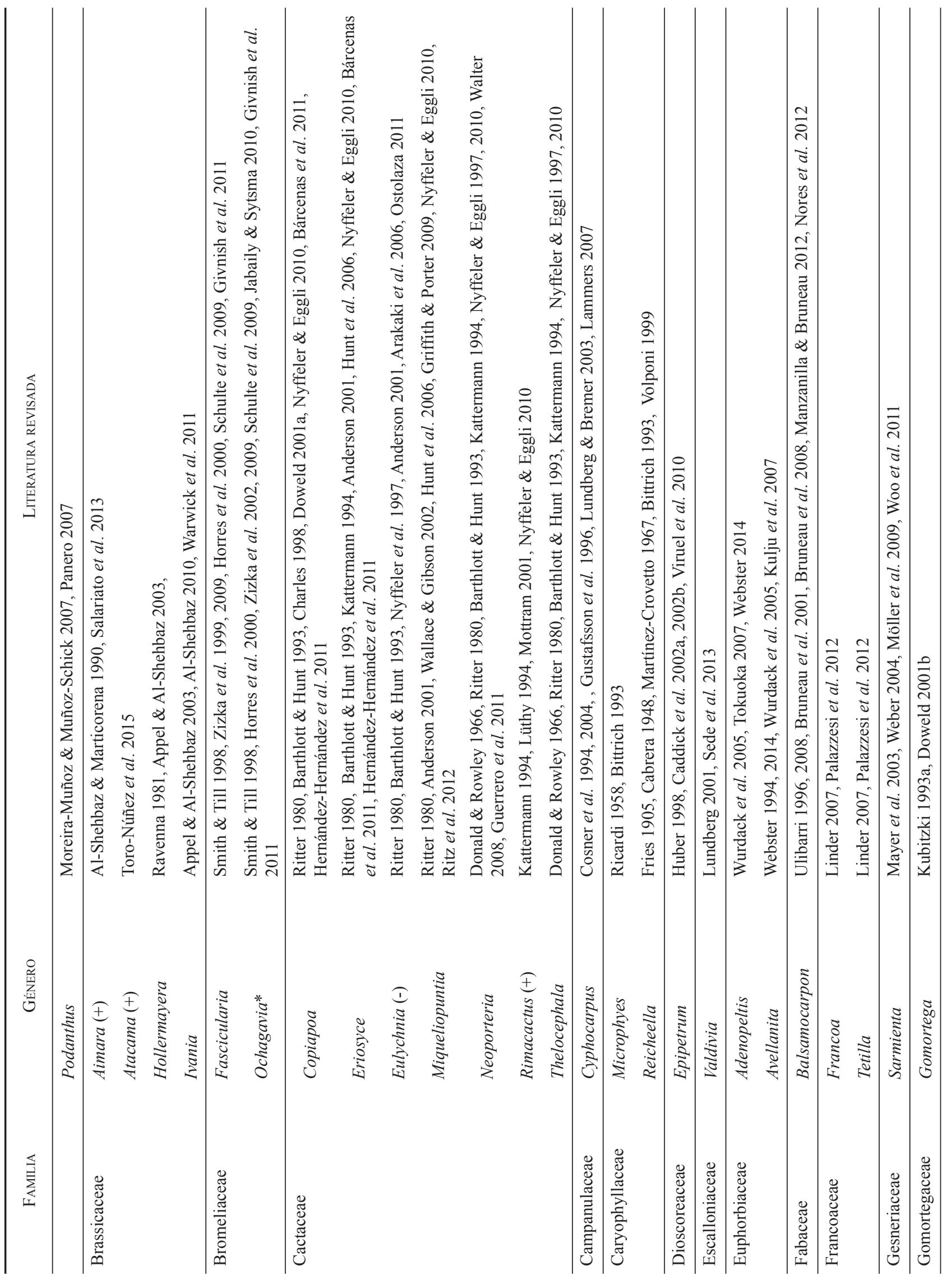




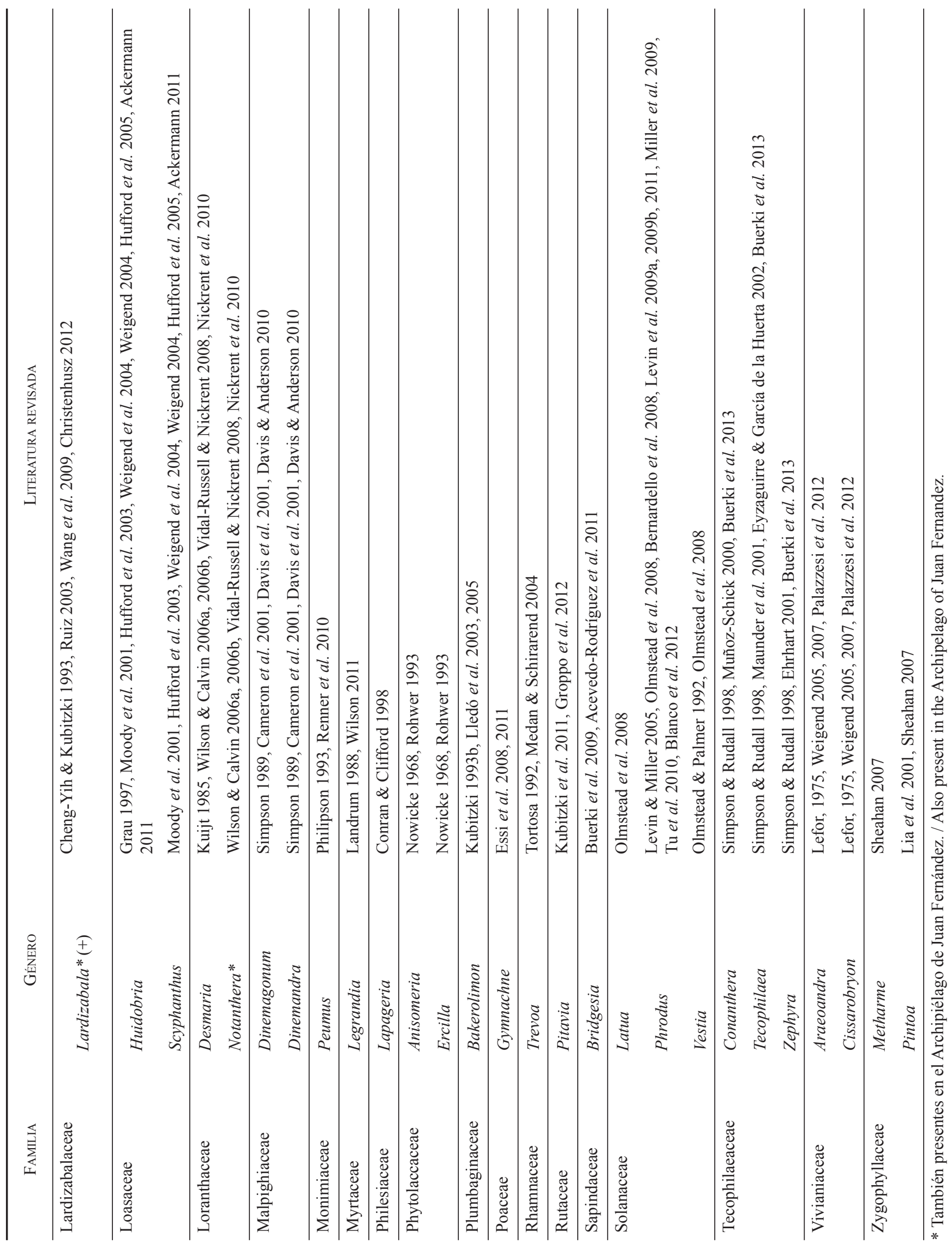


Tabla II. Resumen de los géneros propuestos como endémicos del Archipiélago de Juan Fernández, sobre la base de Moreira-Muñoz (2011), el Catálogo de las Plantas Vasculares del Cono Sur y nuestra revisión bibliográfica. Se indican con signos (+) y (-) las adiciones y las sustracciones resultado de nuestra revisión, respecto a las dos fuentes señaladas anteriormente.

TABle II. Summary of proposed endemic genera of Juan Fernández Archipielago, based on Moreira-Muñoz (2011), the Catalogue of Vascular Plants of the Southern Cone and our bibliographic revision. (+) and (-) indicate additions and substractions to the lists provided by Moreira-Muñoz (2011) and the Catalogue based on our revision.

\begin{tabular}{|c|c|c|}
\hline FAMILIA & GÉNERO & LITERATURA REVISADA \\
\hline Arecaceae & Juania & Trénel et al. 2007 \\
\hline \multirow[t]{4}{*}{ Asteraceae } & Centaurodendron & $\begin{array}{l}\text { Carlquist 1958, Dittrich 1977, Moreira-Muñoz \& Muñoz-Schick 2007, Susanna \& } \\
\text { Garcia-Jacas 2007, Susanna et al. } 2011\end{array}$ \\
\hline & Dendroseris & $\begin{array}{l}\text { Carlquist 1967, Kim et al. 1996, 1999, 2007, Lee et al. 2005, Moreira-Muñoz \& } \\
\text { Muñoz-Schick 2007, Kilian et al. 2009, Mejias \& Kim } 2012\end{array}$ \\
\hline & Robinsonia & Moreira-Muñoz \& Muñoz-Schick 2007, Pelser et al. 2007, 2010 \\
\hline & Yunquea & $\begin{array}{l}\text { Carlquist 1958, Dittrich 1977, Moreira-Muñoz \& Muñoz-Schick 2007, Susanna \& } \\
\text { Garcia-Jacas 2007, Susanna et al. } 2011\end{array}$ \\
\hline Boraginaceae & Selkirkia & Johnston 1927 \\
\hline Lactoridaceae & Lactoris & $\begin{array}{l}\text { Kubitzki 1993c, Sampson 1995, Stuessy et al. 1998, González \& Rudall 2001, } \\
\text { Ricci } 2001\end{array}$ \\
\hline Lamiaceae & Cuminia & Ruiz et al. 2000 \\
\hline Myrtaceae & Nothomyrcia $(+)$ & Wilson 2011, Murillo-Aldana \& Ruiz 2011, Murillo-Aldana et al. 2012, 2013 \\
\hline \multirow[t]{2}{*}{ Poaceae } & Megalachne & Schneider et al. 2011 \\
\hline & Podophorus & Baeza et al. 2002, Schneider et al. 2011 \\
\hline Rosaceae & Margyracaena & Crawford et al. 1993, Kalkman 2004 \\
\hline Thyrsopteridaceae & Thyrsopteris & $\begin{array}{l}\text { Smith et al. 2006, Schuettpelz \& Pryer 2007, Kuo et al. 2011, Lehtonen 2011, } \\
\text { Christenhusz \& Chase } 2014\end{array}$ \\
\hline
\end{tabular}

TABLA III. Resumen de los géneros propuestos como endémicos de las Islas Desventuradas, sobre la base de Moreira-Muñoz (2011), el Catálogo de las Plantas Vasculares del Cono Sur y nuestra revisión bibliográfica. Se indican con signos (+) y (-) las adiciones y las sustracciones resultado de nuestra revisión, respecto a las dos fuentes señaladas anteriormente.

TABLE III. Summary of proposed endemic genera of Desventuradas Islands, based on Moreira-Muñoz (2011), the Catalogue of Vascular Plants of the Southern Cone and our bibliographic revision. (+) and (-) indicate additions and substractions to the lists provided by MoreiraMuñoz (2011) and the Catalogue based on our revision.

\begin{tabular}{lll}
\hline \multirow{2}{*}{ FAmilia } & \multicolumn{1}{c}{ GÉNERo } & \multicolumn{1}{c}{ Literatura REVISADA } \\
\hline Asteraceae & Lycapsus & Robinson 1981, Baldwin et al. 2002, Panero 2007 \\
\cline { 2 - 3 } & Thamnoseris & $\begin{array}{l}\text { Kim } \text { et al. 1996, 1999, 2007, Lee } \text { et al. 2005, Moreira-Muñoz \& Muñoz-Schick } \\
\text { 2007, Kilian } \text { et al. 2009, Mejias \& Kim 2012 }\end{array}$ \\
\hline Boraginaceae & Nesocaryum & Johnston 1927 \\
\hline Caryophyllaceae & Sanctambrosia* & Bittrich 1993 \\
\hline
\end{tabular}

* Actualizado según Muñoz-Schick \& Morales (2013). / Updated according to Muñoz-Schick \& Morales (2013). 
I Géneros endémicos de Chile Continental

\section{Alliaceae}

Ancrumia Harv. ex Baker, Gethyum Phil. y Gilliesia Lindl.

Según Fay \& Hall (2007) y Escobar (2012) existen distintos tratamientos para la tribu Gilliesieae. En algunos de ellos Gethyum, Ancrumia y Solaria han sido tratados por separado (e.g. Marticorena \& Quezada 1985, Fay \& Chase 1996, Chase et al. 2009), en otro Ancrumia ha sido incluido en Gethyum (Muñoz-Schick 2000), y un último donde todos los géneros han sido fusionados en Solaria (Ravenna 2000).

Según Escobar (2012), dentro de la tribu Gilliesieae, Gilliesia, Gethyum y Solaria constituyen un clado bien sustentado estadísticamente. La autora propone la revalidación de Ancrumia como un género basal de este clado, con un único representante anteriormente tratado en Gethyum (A. cuspidata Harv. ex Baker), en base a su androceo de sólo 2 estambres y su distribución geográfica. Considerando este cambio, Gilliesia sería parafilético con Solaria y la otra especie de Gethyum incluidos en él. A pesar de esto, Escobar (2012) propone mantener la actual delimitación de estos últimos tres géneros en favor de la estabilidad taxonómica, señalando que si bien las filogenias moleculares indican parafilia, esto no coincide con los análisis morfológicos. Cabe señalar que el clado GilliesiaGethyum-Solaria está formado por dos clados principales, uno Gilliesia-Solaria y otro Gilliesia-Gethyum, siendo este último clado endémico del país. Por otro lado, el género Gilliesia, que no había sido considerado como endémico en la revisión de Moreira-Muñoz (2011) ni por el Catálogo, se distribuye principalmente en Chile con un único registro en Argentina, en la localidad de Mendoza. Muñoz-Schick (2000) revisó dicho material y la descripción del mismo y coincide en su identificación, señalando entonces que el género no sería endémico del país. No obstante, Escobar (2012) concuerda con Ravenna (2005) y señala que habría un error en la ubicación geográfica que se indica en la etiqueta, ya que no hay otro registro en la Cordillera de los Andes. Escobar (com. pers.) siguió la historia de la colección y concluye que ésta pudo haber ocurrido en Chile a mucho menor altitud. Por lo tanto, Gethyum, Gilliesia y Ancrumia se consideran géneros endémicos de Chile a la espera de una nueva clasificación de los géneros de la tribu.

Miersia Lindl. y Speea Loes.

Según Escobar (2012) Speea se encontraría dentro de Miersia, sin embargo, la autora no propone ningún arreglo taxonómico al respecto siguiendo el mismo criterio señalado anteriormente en Gilliesia-Gethyum-Solaria, por lo que ambos géneros seguirían siendo endémicos de Chile a la espera de una nueva clasificación.
Alstroemeriaceae

Leontochir Phil.

Estudios recientes como Alzate et al. (2008) y Chacon et al. (2012) coinciden en la inclusión de Leontochir en Bomarea, siguiendo la recomendación de Aagesen \& Sanso (2003) sobre la base de caracteres morfológicos, ya que la evidencia cariológica y molecular, si bien pone a ambos géneros en un mismo clado con buen sustento estadístico, no es concluyente respecto a la posición de Leontochir dentro de él (Aagesen \& Sanso 2003, Palma-Rojas et al. 2007, Alzate et al. 2008, Chacon et al. 2012, Petersen et al. 2013). Aagesen \& Sanso (2003) proponen una filogenia donde Leontochir aparece como el taxon basal del clado Leontochir-Bomarea, mientras que en las filogenias de Chacón et al. (2012) y Petersen et al. (2013) no hay resolución dentro de éste. Por lo tanto se propone conservar el estatus de Leontochir como género endémico de Chile a la espera de una mejor resolución filogenética que permita establecer su posición en el clado, sobre la cual discutir morfológicamente la interpretación de la filogenia.

\section{AMARYLLIDACEAE}

Famatina Ravenna, Phycella Lindl., Placea Miers y Traubia Moldenke

La clasificación de la tribu Hippeastreae ha sido objeto de controversia debido a que no se han identificado suficientes caracteres diagnósticos y a la homoplasía (García et al. 2014). Los mismos autores proponen dos subtribus fundamentadas sobre la base de análisis moleculares; una de ellas, Traubiinae, está formada casi exclusivamente por especies endémicas de Chile, exceptuando dos de distribución marginal en Argentina, ambas incluidas en Rhodolirium. Los análisis realizados por García et al. (2014) señalan que Traubia, el único género monotípico del grupo, sería un taxón basal de la subtribu. Respecto de los géneros no monotípicos, Phycella, Placea y Rhodolirium ponen en duda su estatus actual por ser parafiléticos en esta filogenia. De las especies que forman Famatina sólo F. maulensis Ravenna estaría claramente incluida dentro de este clado. Phycella, a su vez, no había sido considerado como un género endémico por la presencia de $P$. herbertiana Lindl. en Argentina, sin embargo, dicha especie, tratada también como Famatina, se encontraría ubicada lejos de Phycella s.s., en Hippeastrinae (García et al. 2014). En consecuencia, Phycella, Placea y Traubia serían géneros endémicos de Chile, a la espera de una nueva delimitación genérica, siendo Traubia la propuesta más estable por ser monotípico y basal. García et al. (2014) señalan que la resolución de sus análisis no es suficiente para proponer una nueva clasificación genérica, pero ciertamente ponen en duda la actualmente utilizada. El Catálogo considera a Famatina como un género con dos especies, ambas endémicas de Chile; sin embargo, la parafilia demostrada por García et al. (2014) y la inclusión de F. herbertiana 
(Lindl.) Ravenna deja profundamente cuestionada su existencia, circunscripción y endemismo respecto a Chile, por lo que se propone descartarlo como género endémico de Chile. Finalmente, se señala que Placea no cuenta con un número claro de especies, el que varía entre cinco y siete (Guerrero et al. 2007, Cisternas et al. 2010 y García et al. 2014); P. germainii Phil. y P. grandiflora Lem. son los taxa más dudosos, los que según Muñoz-Schick (2000) serían sinónimos de $P$. ornata Miers ex Lindl.

\section{Apiaceae}

Homalocarpus Hook. \& Arn.

Schwarzer et al. (2010) han citado recientemente a $H$. digitatus (Phil.) Math. \& Const. para el desierto de Perú lo que implica que dejaría de ser considerado como endémico de Chile.

\section{Asteraceae}

\section{Calopappus Meyen}

Género monotípico aparentemente hermano del grupo Nassauvia-Triptilion. Las filogenias moleculares de Simpson et al. (2009) y Maraner et al. (2012) corroboran su estatus de género, a pesar de que el análisis cladístico morfológico previo de Freire et al. (1993) no coincide e incluye al género en Nassauvia. Morfológicamente, Hind (2007) y Bremer (1994 fide Katinas et al. 2008a) consideran al género como parte de Nassauvia, sin embargo, Katinas et al. (2008a) revalidan el género en su revisión. La filogenia molecular de Simpson et al. (2009) propone con fundamento a Calopappus como hermano de Nassauvia-Triptilion, sin embargo, en la de Maraner et al. (2012) que incluye un muestreo con más especies de Nassauvia, el sustento estadístico disminuye considerablemente; ninguna de las dos cuestiona el estatus de género a Calopappus, ya que en ambas queda como grupo hermano de Nassauvia-Triptilion. Por lo tanto, a diferencia de lo que sostienen Freire et al. (1993), Nassauvia dejaría de ser parafilético si se incorporara a Triptilion y no a Calopappus. De esta manera, se propone mantener a Calopappus como género endémico a la espera de una mejor resolución filogenética del grupo.

Kieslingia Faúndez, Saldivia \& A.E. Martic.

Género recientemente descrito sobre la base de una única especie, Kieslingia chilensis Faúndez, Saldivia \& A.E. Martic. (Saldivia et al. 2014). En base al análisis molecular, Kieslingia es género hermano de Guynesomia Bonifacino \& Sancho (también endémico de Chile), sin embargo, características morfológicas como tipo de capítulo, sinflorescencia y ápice de las hojas, sustentan la propuesta de un nuevo género (Saldivia et al. 2014).

BRASSICACEAE

Aimara Salariato \& Al-Shehbaz

Salariato et al. (2013) llevaron a cabo un análisis molecular del género Menonvillea DC. y géneros relacionados, y reportaron la segregación de la especie $M$. rollinsii AlShehbaz \& Martic., para la que propusieron la creación de un género monotípico nuevo para Chile, Aimara rollinsii (Al-Shehbaz \& Martic.) Salariato \& Al-Shehbaz, delimitado también sobre la base de algunos antecedentes morfológicos, principalmente de su hábito leñoso único dentro de Menonvillea (Al-Shehbaz \& Marticorena 1990).

\section{Atacama Toro, Mort \& Al-Shehbaz}

Toro-Núñez et al. (2015) realizaron análisis moleculares y de nicho del género Mathewsia Hook. \& Arn., segregando a la especie $M$. nivea (Phil.) O.E.Schulz en un nuevo género endémico para Chile llamado Atacama, bajo el nombre de $A$. nivea (Phil.) Toro, Mort \& Al-Shehbaz. Morfológicamente, Atacama se diferencia en sus hojas, frutos y pétalos. Sin embargo, esos caracteres exhiben alto grado de plasticidad fenotípica, lo que no permite una clara discriminación taxonómica. No obstante, tanto los resultados filogenéticos como el análisis de nicho ecológico la distinguen claramente de Mathewsia."

\section{Bromeliaceae}

\section{Fascicularia Mez y Ochagavia Phil.}

La mayoría de los estudios filogenéticos recientes muestran a ambos géneros como un clado bien soportado (Horres et al. 2000, Schulte et al. 2009, Givinish et al. 2011) en el que aparentemente el género Fascicularia estaría incluido dentro de Ochagavia (Givinish et al. 2011). Sin embargo, los resultados de estos análisis no se consideran concluyentes por el reducido muestreo de taxa de Ochagavia, cuyas especies no siempre aparecen como un grupo monofilético (Jabaily \& Sytsma 2010). Por lo tanto proponemos dejar ambos géneros hasta contar con una filogenia con un mejor muestreo de taxa y una mejor resolución.

\section{Cactaceae}

Eriosyce sensu stricto Phil.

Kattermann (1994) incluye en Eriosyce a Islaya (Chile y Perú), Pyrrhocactus s.s. (Argentina), Neoporteria, Horridocactus y Thelocephala (exclusivos de Chile), los que anteriormente habían sido agrupados en Neoporteria (Donald \& Rowley 1966). El criterio ampliado de Eriosyce s.l. planteado por Kattermann (1994) ha sido ampliamente utilizado en la literatura sobre cactáceas (e.g. Anderson 2001, Hoffmann \& Walter 2004, Hunt et al. 2006) convirtiéndose en una propuesta estable hasta los años recientes. Dicha propuesta, sugerida con anterioridad por Hunt \& Taylor (1986), fue establecida según el análisis filogenético basado en morfología de Wallace (1994), separando el género Eriosyce s.l. en dos secciones, divididas a su vez en tres subsecciones cada una. Este esquema conserva mayoritariamente la propuesta de géneros de Ritter (1980) para Chile, pero en niveles jerárquicos 
inferiores. No obstante, Kiesling et al. (2008) no reconocen la propuesta de Kattermann (1994), y sostienen el esquema de Ritter (1980) con Islaya, Neoporteria, Pyrrhocactus (incluido Horridocactus) y Thelocephala como géneros independientes, criterio que ha sido seguido por MoreiraMuñoz (2011).

Recientes estudios moleculares (Nyffeler \& Eggli 2010, Bárcenas et al. 2011, Hernández-Hernández et al. 2011) sugieren que Eriosyce s.l. sería un género parafilético, aunque sin relaciones resueltas dentro de éste. Por otro lado, Eriosyce s.s. es un género endémico de Chile, cuyos atributos morfológicos como el indumento y espiniscencia del fruto, tamaño de los cuerpos y las semillas, lo separan claramente del resto de los géneros señalados, por lo que se propone mantener la circunscripción génerica en el sentido estricto.

\section{Eulychnia Phil.}

Género de distribución mayoritariamente chilena, principalmente en la costa, desde el sur de Arica hasta la Región de Valparaíso. Si bien se lo ha señalado como endémico de Chile (Moreira-Muñoz 2011), E. ritteri Cullmann es una especie endémica de la costa sur de Perú disyunta con el resto de las especies de Chile (Nyffeler et al. 1997, Anderson 2001, Arakaki et al. 2006, Ostolaza 2011).

\section{Miqueliopuntia Frič ex F.Ritter}

Género monotípico creado por Friedrich Ritter en 1980. Kiesling et al. (2008) lo consideran sinónimo de Austrocylindropuntia siguiendo el criterio de Backeberg (1958), sin embargo, tratamientos recientes de la familia lo consideran como un género válido (Anderson 2001, Wallace \& Gibson 2002, Hunt et al. 2006). Por otro lado, Griffith \& Porter (2009) sobre la base de análisis moleculares confirman el criterio de Ritter (1980), que propone a Miqueliopuntia como un género independiente de Austrocylindropuntia y más cercano a Airampoa (Tunilla), lo que concuerda además con sus distribuciones geográficas (Ritz et al. 2012). Actualmente ambos géneros se consideran miembros de subtribus distintas, Austrocylindropuntia en Cylindropuntieae y Miqueliopuntia en Opuntieae (Nyffeler \& Eggli 2010), por lo tanto, corresponde mantener el estatus de género monotípico y endémico de Chile.

\section{Neoporteria Britton \& Rose}

El género Neoporteria, creado por Britton \& Rose (1922), fue correctamente circunscrito por Backeberg (1959) y Ritter (1980), basado en sus características florales, tanto morfológicas como funcionales (polinización). Con excepción de Donald \& Rowley (1966), Neoporteria ha sido considerado como grupo natural por distintos autores, independiente de su rango taxonómico (Walter 2008, Guerrero et al. 2011), sin embargo, la propuesta de Kattermann (1994) que incluye a Neoporteria en Eriosyce s.l. como taxón infragenérico no ha sido objetada salvo por Kiesling et al. (2008) en donde se mantiene al nivel de género.

Estudios de diversa índole sugieren la monofilia de Neoporteria s.s. independiente de su rango taxonómico, ya sea sobre la base de caracteres anatómicos del tallo (Nyffeler \& Eggli 1997), patrones de floración y morfología floral (Walter 2008) o morfología y análisis filogenéticos moleculares (Guerrero et al. 2011). Sin embargo, en todos estos trabajos se discute la inclusión o exclusión de especies intermedias con Pyrrhocactus s.l. (e.g. P. chilensis, P. taltalensis, Neoporteria sociabilis) para establecer la correcta monofilia del grupo. No obstante estos cambios, Neoporteria sigue siendo un grupo endémico de Chile.

Ante la evidencia del origen no monofilético de Eriosyce s.l. (Nyffeler \& Eggli 2010, Bárcenas et al. 2011, HernándezHernández et al. 2011), consideramos mantener el estatus genérico de Neoporteria y su condición de endémico para Chile.

\section{Rimacactus Mottram}

Lüthy (1994) describe a Eriosyce laui dentro del trabajo de Kattermann (1994), una singular nueva especie con una combinación de atributos morfológicos difícil de interpretar. Kattermann (1994) la ubica dentro de la subsección Chileosyce, aún cuando Lüthy indica que su posición taxonómica debería estar en Islaya o en la subsección Islaya en el sentido de Kattermann (1994), o incluso en Copiapoa (Lüthy 1994).

Mottram (2001), basándose en los atributos morfológicos y experimentos de polinización, propone a Rimacactus como un nuevo género para $E$. laui, ubicándolo cercano a los géneros Matucana y Borzicactus (tribu Cereeae), y alejado de Eriosyce s.l. (tribu Notocacteae). Nyffeler \& Eggli (2010), sobre la base de análisis moleculares, confirman la categoría de género monotípico de Mottram (2001), ubicando a Rimacactus en un clado de la tribu Notocacteae relacionado con los géneros Yavia (norte de Argentina) y Neowerdermannia (Norte de Chile y Argentina, y sur de Perú y Bolivia) y no con Eriosyce s.l.

\section{Thelocephala Y. Ito}

El género Thelocephala, enmendado y revisado por Ritter (1959, 1980), comprende a cactáceas pigmeas, geófitas, con dispersión anemócora de sus frutos. Kattermann (1994) lo incluye en Eriosyce s.l. en dos subsecciones, Horridocactus (incluido el tipo, T. napina) y Chileosyce, proponiendo a este último como un nuevo taxón creado para agrupar a los taxa con frutos cubiertos de cerdas rígidas, lo que implicaría de forma más estricta la dispersión por el viento, que lo planteado por Ritter (1980).

Nyffeler \& Eggli (1997) en consideración de la anatomía del tallo, validan la propuesta de Ritter (1980) de Thelocephala por sobre la de Kattermann (1994) de Chileosyce. Si bien, los análisis moleculares posteriores de 
Nyffeler \& Eggli (2010) indican un bajo sustento estadístico para el concepto ampliado de Eriosyce s.l. (Kattermann 1994), también muestran relaciones no resueltas entre especies de Thelocephala y Pyrrocactus de Chile (= Horridocactus), aunque con pocas especies incluidas en el análisis. Sin duda, Thelocephala y Pyrrhocactus de Chile son muy cercanos (Ritter 1980) por lo que es necesario un análisis más robusto en términos de número de especies para dilucidar dichas relaciones y establecer correctamente las circunscripciones genéricas.

\section{Caryophyllaceae}

\section{Reicheella Pax}

Reicheella es un género monotípico señalado como endémico de Chile (Zuloaga et al. 2008, Moreira-Muñoz 2011), a pesar que Fries (1905) señala su presencia en la alta cordillera de la provincia de Jujuy en Argentina, referencia aceptada por Martínez-Crovetto (1967). Volponi (1999), sin embargo, lo considera como una referencia dudosa ya que los materiales no habrían sido revisados por especialistas.

Los materiales citados por Fries corresponden a: ARGENTINA, Puna de Jujuy, Nevado de Chañi, ca. 4.500 m, 28 - XI - 1901, R.E. Fries 863, S (S06-7229), UPS (UPS: BOT: V-201362).

Éstos fueron revisados por C.R. Volponi (com. pers.), confirmando que no corresponden a Reicheella. Esta situación reafirma lo expuesto por Cabrera (1948), quien no señala la presencia de Reicheella para la Puna Argentina. Además A.L. Cabrera en sus viajes de recolección de materiales para la publicación de la "Flora de Jujuy" visitó el Nevado de Chañi en varias oportunidades sin coleccionar ejemplares de Reicheella (C.R. Volponi, com. pers.). En consecuencia, se confirma a Reicheella como un género endémico de Chile.

\section{DiosCoreaceae}

\section{Epipetrum Phil.}

El Catálogo acepta la reclasificación de Caddick et al. (2002a) que incluye a las especies de Epipetrum en Dioscorea sobre la base de análisis filogenéticos combinados de morfología y datos moleculares de la familia Dioscoreaceae (Caddick et al. 2002b); sin embargo, para ese análisis sólo se contó con datos morfológicos y no moleculares de una sola de las tres especies de Epipetrum reconocidas. El árbol filogenético de evidencia total, que incluye tanto los resultados de la morfología como los datos moleculares, muestra un clado bien sustentado estadísticamente en el que se encuentran algunos de los miembros del grupo que hoy se incluyen en Dioscorea. Si bien las especies de Epipetrum no se encuentran en este árbol, son consideradas como Dioscorea por sus afinidades con los demás miembros del clado y por su posición en el árbol morfológico, aunque existe un bajo sustento estadístico para ello. Viruel et al. (2010), en su reciente revisión sistemática del grupo Epipetrum, indican que el número de cromosomas es una interesante sinapomorfia para este grupo, haciéndolo diferente de las otras especies de Dioscoreaceae. Se decide por lo tanto dejar a Epipetrum como género endémico hasta que se completen las filogenias con los datos moleculares necesarios, ya que los datos morfológicos y cariológicos no son aún concluyentes.

\section{EUPHORBIACEAE}

Adenopeltis Bertero ex A. Juss.

Las filogenias disponibles (Wurdack et al. 2005, Tokuoka 2007) no logran buena resolución en la tribu Hippomaneae subtribu Hippomaninae, observándose parafilia en varios géneros hermanos, lo que sugiere la necesidad de revisar la delimitación genérica en la subtribu (Wurdack et al. 2005, Tokuoka 2007). Webster (2014) señala, a su vez, que la inclusión de Adenopeltis y Spegazziniophytum en Stillingia debe ser considerada seriamente, y que no está formalmente propuesta en su revisión sólo porque las nuevas combinaciones no se encuentran aún disponibles. En consecuencia, se propone conservar su estatus de género endémico en espera de una mejor resolución filogenética para la subtribu, que permita o no sustentar la hipótesis de Webster (2014).

\section{Balsamocarpon Clos}

Las relaciones del género no están sustentadas filogenéticamente. Ulibarri (1996) lo ubica al interior de Caesalpinia siguiendo el criterio de Reiche, sin embargo, posteriormente vuelve a considerarlo como un género endémico de Chile dentro de Caesalpinioideae (Ulibarri 2008), grupo que actualmente no cuenta con una filogenia ni una delimitación genérica claras (Manzanilla \& Bruneau 2012, Nores et al. 2012, Bruneau et al. 2008, Bruneau et al. 2001). Por lo tanto, se propone conservar su estatus de género a la espera de una mejor resolución filogenética que ayude a la comprensión del clado.

\section{LARDIZABALACEAE}

\section{Lardizabala Ruiz \& Pav.}

Moreira-Muñoz (2011) no considera a este género como endémico. Ruiz (2003) y Christenhusz (2012) proponen que el género tendría distribución en "Argentina adyacente", sin embargo, todos los registros citados y encontrados corresponden a Chile. Zuloaga \& Morrone (1999), Zuloaga et al. (1999) y el Catálogo no consideran registros en Argentina, de modo que se propone darle el estatus de género endémico del país. Por otro lado, cabe señalar que la presencia del género en Juan Fernández se debería a una introducción desde el continente (Marticorena 1990, Danton et al. 2006). 
LOASACEAE

\section{Huidobria Gay}

A pesar de ser reconocido como género endémico por Moreira-Muñoz (2011) y el Catálogo, Huidobria aparece como parafilético en varios análisis filogenéticos con ambas especies en una politomía en la base de la subfamilia Loasoideae (Moody et al. 2001, Hufford et al. 2003, Weigend et al. 2004, Hufford et al. 2005). Sin embargo, Hufford et al. (2005) señalan que esto requiere pruebas y datos filogenéticos adicionales. Por lo tanto, se propone conservar su estatus de género endémico a la espera de una mejor resolución filogenética.

\section{Scyphanthus Sweet}

Género con una o dos especies (Weigend et al. 2004, Ackermann 2011) y de posición no resuelta dentro de la filogenia de Loasaceae. En la mayoría de los casos se presenta inlcuido en Loasa, junto con Caiophora y Blumenbachia (Moody et al. 2001, Hufford et al. 2003, Weigend et al. 2004, Hufford et al. 2005), lo que podría provocar una futura reclasificación. Respecto de su distribución geográfica, Scyphanthus ha sido considerado como endémico de Chile (Marticorena 1990, Zuloaga et al. 2008, Moreira-Muñoz 2011). No obstante, existen cinco colecciones de herbario que señalan su presencia en Argentina:

ARGENTINA, prov. de Neuquén, San Carlos de Bariloche, lago Nahuelhuapi, $41^{\circ}$ s. Br., In Gebüschen der Pampas, 800 m, 11 - II - 1905, O. Buchtien s/n, E (277489, 277488).

ARGENTINA, Patagonia, Nahuelhuapi, VI - 1906, M. Gandoger s/n, MO (5468170).

ARGENTINA, Sta. Cruz, Tehuelches, 250 m, 19 - XI 1928, A. Donat 3, MO (979113).

ARGENTINA, Mendoza, 1826, sin colector, K (372955).

Los materiales fueron revisados por M. Weigend (com. pers.), encontrándose que los de Edinburgo (E) corresponden en realidad a Loasa bergii Hieron. y los de Missouri (MO), a Loasa filicifolia Poepp. El ejemplar de Kew (K) está correctamente determinado como Scyphanthus stenocarpus Urb. \& Gilg, sin embargo, la carpeta tiene dos etiquetas adicionales, una de Sleumer (1953) que indica "False ticket: Scyphanthus does not occur in Argentina", y otra anónima que alude a lo mismo "right ticket?". Por lo tanto, en vista de la fecha de la colección (1826) y de que no se han hecho nuevas colecciones del género en Argentina, se propone mantener el estatus de género endémico de Chile y la presencia en Argentina como dudosa, debiendo ser corroborada con más colecciones.

\section{Plumbaginaceae}

Bakerolimon O.A. Lincz.

Según Lledó et al. (2005), Linczevski, autor del género, señala que el género habitaría las regiones desérticas no sólo de Chile, sino también de Perú; lo que concuerda con dos ejemplares de herbario provenientes de Perú y depositados en el herbario de Kew correspondientes al isotipo y tipo de B. peruvianum (Kuntze) Lincz.:

PERÚ, McLean s/n, K (572629 holotipo, 572628 isotipo). Sin embargo, Lledó et al. (2003) señalan que B. peruvianum habría sido descrito en territorios antiguamente peruanos y actualmente chilenos. Por otro lado, Bakerolimon fue agregado a la Flora de Perú por Ulloa et al. (2004), basándose en la descripción original del género, y no en nuevas colecciones. Por lo tanto, se propone conservar su estatus de género endémico y dejar como dudosa su presencia en Perú.

PoAceAe

\section{Gymnachne Parodi}

El Catálogo sigue la nomenclatura propuesta por Essi et al. (2011) en la que el clado de especies americanas de Briza s.l., incluido Gymnachne, fue renombrado como Chascolytrum sobre la base de un análisis filogenético previo (Essi et al. 2008). Sin embargo, la posición de Gymnachne en el complejo Briza no está resuelta, ya que el soporte estadístico del árbol es bajo, por lo que no se puede descartar que Gymnachne sea un género hermano del resto de Chascolytrum. Por lo tanto, se propone conservar el estatus de género endémico de Chile a la espera de una filogenia mejor resuelta.

\section{Solanaceae}

\section{Phrodus Miers}

El Catálogo adscribe a la inclusión de Phrodus en Lycium, propuesta por Levin et al. (2011), basados en análisis filogenéticos, morfológicos y de cariotipo. En la filogenia, Phrodus aparece en la base del clado Lycium-Grabowskia, con sustento estadístico moderado (Miller et al. 2009, Levin et al. 2011). Si bien anteriormente análisis con marcadores plastidiales señalaban que estaría incluido al interior de Lycium con bajo soporte estadístico (Levin \& Miller 2005, Levin et al. 2009a, 2009b, Olmstead et al. 2008), su cariotipo coincide con características basales de la tribu Lycieae y se distingue de las otras especies de Lycium (Bernardello et al. 2008, Blanco et al. 2012). Los autores coinciden en que la filogenia no es concluyente para incluir a Phrodus en Lycium, sin embargo, tampoco es contradictoria, de manera que se decide incluir a Phrodus en Lycium por ser monotípico el primero y más rico en especies el segundo, señalando también que las características morfológicas utilizadas para distinguir al género se encuentran también en Lycium y podrían ser simplesiomórficas (Levin et al. 2011). Sin embargo, se propone conservar el estatus del género a la espera de una mejoría en la resolución de la base de la tribu.

\section{Vestia Willd.}

Vestia es un género monotípico de Solanaceae (Cestroideae: Cestreae) relacionado con Cestrum y Sessea (Olmstead \& Palmer 1992, Olmstead et al. 2008), considerado endémico del centro-sur de Chile (Marticorena 1990, Zuloaga et al. 
2008, Moreira-Muñoz 2011). Sin embargo, en el Herbario Nacional de Paris (P) se conservan cinco colecciones de Vestia cuyas etiquetas señalan como origen Perú:

PERÚ Y CHILE, Herb. Richard s/n, P (454648).

PERÚ, J. Dombey s/n, P (454649).

PERÚ, Leman s/n, P (454650).

PERÚ, Schl. s/n, P (454651).

PERÚ, CHILE, J. Dombey s/n, P (454652).

Estas colecciones no tienen fecha, pero al menos dos de ellas $(454649,454652)$ señalan como su colector a J. Dombey, por lo que se asume que se trata de materiales antiguos, ya que H. Ruiz, J. Pavón y J. Dombey recorrieron y colectaron intensamente en Perú y Chile entre 1778 y 1783 (Marticorena, 1995). Por otro lado, existe una colección de Vestia en París (J. Dombey 383, 454653) asignada a Concepción, con el mismo tipo de etiqueta e idéntica grafía original que 454652 . Dos colecciones $(454648,454652)$ señalan ambiguamente como sitio de colección a ambos países, por lo que es posible que exista una confusión en el etiquetado original. Por otro lado, Vestia no ha sido considerado para la flora de Perú (Brako \& Zarucchi 1993, Ulloa et al. 2004, Rodríguez et al. 2006) y según su distribución conocida, resulta poco probable su presencia en ese país. Por lo tanto, se conserva el estatus de género endémico y se deja dudosa la presencia del género en Perú.

\section{TeCophileaceae}

Zephyra D. Don y Tecophilaea Bert. ex Colla Buerki et al. (2013) analizan la relación entre Zephyra y Tecophilaea bajo el concepto de Ravenna (1998), quien incluye a $T$. cyanocrocus y $T$. violiflora en Zephyra; dicho análisis muestra a Z. elegans incluida en Tecophilaea sin soporte estadístico, formando ambos géneros un grupo monofilético bien sustentado. Por otro lado, Zuloaga et al. (2008) reconoce una nueva especie descrita por Ehrhart (2001) (Z. compacta C. Ehrh.) no considerada en la filogenia de Buerki et al. (2013). En consecuencia, si bien es probable que ambos géneros se fusionen, es necesaria la inclusión de esta nueva especie en los análisis para descartar una relación de grupos hermanos.

\section{ViviANIACEAE}

Araeoandra Lefor y Cissarobryon Lefor

Según Weigend (2005) existen dos tratamientos para los géneros de Vivianiaceae. Uno tradicional que incluye a Araeoandra, Caesarea y Cissarobryon en Viviania, y otro que los separa (Lefor, 1975). Weigend (2007) incorpora a Vivianiaceae en Ledocarpaceae y señala que la subdivisión propuesta por Lefor (1975) no tendría justificación. El autor argumenta que si bien los géneros por separado pueden o no ser grupos naturales, Viviania s.l. sí lo es y, sin señalar aspectos morfoanatómicos o moleculares, no ve ningún motivo para su separación dado que el grupo contiene apenas seis especies. Palazzesi et al. (2012) proponen una filogenia molecular del grupo que no incluye a Araeoandra, y señalan con buen sustento estadístico que Viviania s.l. es monofilética, sin embargo, también sustenta la subdivisión del grupo en dos clados hermanos: uno con Viviania s.s. por un lado y con Caesarea y Cissarobryon por otro, lo que concuerda también con la división propuesta por Lefor (1975). No obstante, aún es necesaria la introducción de Araeoandra en la filogenia y la discusión de las sinapomorfias que permitan la correcta delimitación del o de los grupos, por lo que se propone conservar el estatus de ambos géneros hasta que se resuelvan dichos puntos.

II Géneros endémicos del Archipiélago de Juan FernándeZ E Islas Desventuradas

\section{Asteraceae}

Centaurodendron Johow y Yunquea Skottsb.

La delimitación de estos géneros ha sido fuente de controversia. Carlquist (1958), sobre la base de estudios anatómicos de ambos géneros, acepta la propuesta de Skottsberg de tratar a ambos géneros por separado. Sin embargo, recientemente el género monotípico Yunquea ha sido tratado como sinónimo de Centaurodendron (Susanna \& Garcia-Jacas 2007, Susanna et al. 2011) sobre la base del estudio de Dittrich (1977), sin integrar a Y. tenzii dentro de los análisis moleculares (Susanna et al. 2011). No obstante, Dittrich (1977) sólo señala con dudas esta posible sinonimia, sin entregar mayores antecedentes. Por lo tanto, ambos géneros se consideran endémicos de Chile a la espera de la inclusión de Yunquea en los análisis moleculares.

Dendroseris D. Don y Thamnoseris F. Phil.

Mejías \& Kim (2012) proponen la incorporación de los géneros Thamnoseris y Dendroseris como un subgénero de Sonchus, a partir de análisis moleculares (Kim et al. 1996, 1999, 2007, Lee et al. 2005) que incluyen a Dendroseris y otros géneros oceánicos dentro de Sonchus. En los análisis moleculares Thamnoseris no fue muestreado, sin embargo Kilian et al. (2009) señalan, sobre la base de una comunicación personal, que la inclusión de Thamnoseris en Sonchus habría sido confirmada por análisis moleculares. Por su parte, la relación entre Dendroseris y Sonchus no está bien sustentada estadísticamente en ninguna filogenia hasta el momento, no siendo posible descartar una relación de grupo hermano entre estos géneros. Por lo tanto, se sugiere conservar a ambos géneros separados de Sonchus a la espera de un aumento en la resolución de los análisis moleculares.

\section{Robinsonia DC.}

El Catálogo incluye a Robinsonia dentro de Senecio, como sugieren Pelser et al. $(2007,2010)$, en base a análisis moleculares, sin embargo, dicha filogenia presenta bajo sustento estadístico. Además, los autores ponen en duda la monofilia del grupo a partir de análisis de secuencias de 
ITS-ETS que con muy baja resolución incluyen a $R$. berteroi en un clado de Senecio continental, separado de las otras Robinsonia de las islas (Pelser et al. 2010). Por otro lado, Takayama et al. $(2013,2014)$ aún reconocen la validez del género argumentando que morfológica y reproductivamente difiere notablemente de Senecio. Sin embargo, sus estudios no tienen relación con la sistemática filogenética del grupo. Si bien es probable la inclusión de Robinsonia en Senecio, el sustento de las filogenias disponibles no permite concluir con certeza la posición del género. Por lo tanto, se propone conservar su estatus a la espera de una mejora en la resolución de las filogenias. A pesar de los bajos soportes de la filogenia, y dada la extensión del muestreo de taxa, creemos que es poco probable que Robinsonia no se encuentre encestado al interior de Senecio. Sin embargo, para ser consistente con los criterios establecidos en este trabajo, hemos querido señalar que dicha filogenia aún necesita un incremento en los soportes para establecer las relaciones al interior del grupo con certeza.

\section{Myrtaceae}

\section{Nothomyrcia Kausel}

Género revalidado (segregado de Myrcengenia) por Murillo-Aldana \& Ruiz (2011), y posteriormente analizado molecularmente, ubicándose filogenéticamente lejos de Myrceugenia, y directamente relacionado con Blepharocalyx (Murillo-Aldana et al. 2012, 2013).

\section{PoAceae}

Megalachne Steud. y Podophorus Phil.

Según Schneider et al. (2011) ambos géneros forman un clado, con Podophorus aparentemente anidado en Megalachne con un soporte estadístico que no es concluyente. Sin embargo, cabe señalar que Baeza et al. (2002) consideran a Podophorus bromoides como "probablemente extinta" y recientemente la especie ha sido clasificada en categoría "extinta" por el sistema nacional de clasificación de especies (D.S. 33/2012, Ministerio del Medio Ambiente, 2012).

\section{Rosaceae}

\section{Margyracaena Bitter}

Históricamente ha sido considerado un género monotípico endémico del archipiélago de Juan Fernández, a pesar de corresponder a un híbrido intergenérico entre Margyricarpus digynus (Bitter) Skottsb. y la especie Acaena argentea Ruiz et Pavon introducida en el archipiélago (Crawford $e t$ al. 1993). Evolutivamente la hibridación ha sido señalada como un importante motor de especiación en plantas (Soltis \& Soltis 2009), de modo que su cualidad de híbrido no debería cuestionar su validez como entidad biológica. Sin embargo, se desconoce si la especie es capaz de reproducirse sexualmente, ya que hasta el momento sólo se ha visto reproducción vegetativa por rizomas (Crawford et al. 1993), lo cual sería un antecedente clave para esta discusión. En consecuencia, se propone conservar su estatus.

\section{AGRADECIMIENTOS}

A los especialistas consultados respecto a los géneros en cuestión. Inelia Escobar (Gilliesia), Lola Lledó (Bakerolimon), Carola Volponi (Reicheella), Carmen Benítez (Vestia) y Maximilian Weigend (Scyphantus). También a James Solomon por el envío de materiales digitales de Scyphanthus en MO, a Mats Hjertson por el envío de materiales digitales de Reicheella en UPS, a Jens Klackenberg por el envío de los mismos en $\mathrm{S}$ y a Rodrigo Flores por el envío de literatura. Agradecemos también a Andrés Moreira y Sebastián Teillier por sus comentarios para mejorar este trabajo. Este estudio fue financiado por el proyecto Fondecyt 11121579 a RS.

\section{BIBLIOGRAFÍA}

Aagesen, L. \& A.M. Sanso. 2003. The Phylogeny of the Alstroemeriaceae, Based on Morphology, rps16 Intron, and rbcL Sequence Data. Systematic Botany 28(1): 47-69.

Acevedo-Rodríguez, P., P.C. Van Welzen, F. Adema \& R.W.J.M. Van Der Ham. 2011. Sapindaceae. In: K. Kubitzki (ed.), The Families and Genera of Vascular Plants, Vol. 10, pp. 357-407. Springer, New York.

Ackermann, M. 2011. Studies on systematics, morphology and taxonomy of Caiophora and reproductive biology of Loasaceae and Mimulus (Phrymaceae). Dissertation zur Erlangung des akademischen Grades des Doktors der Naturwissenschaften (Dr. rer. nat.) eingereicht im Fachbereich Biologie, Chemie, Pharmazie der Freien Universität Berlin, Berlin. 297 pp.

Al-Shehbaz, I.A. 2010. Ivania juncalensis, A Second Species of the Chilean Endemic Ivania (Brassicaceae). Harvard Papers in Botany 15(2): 343-345.

Al-Shehbaz, I.A. \& C. Marticorena. 1990. Menonvillea rollinsii (Brassicaceae), a new shrubby species from Chile. Journal of the Arnold Arboretum 71: 135-138.

Alzate, F., M.E. Mort \& M. Ramírez. 2008. Phylogenetic analyses of Bomarea (Alstroemeriaceae) based on combined analyses of nrDNA ITS, psbA-trnH, rpoB-trnC and matK sequences. Taxon 57(3): 853-862.

Anderson, E.F. 2001. The Cactus Family. Timber Press, Oregon. $776 \mathrm{pp}$.

APG III. 2009. An update of the Angiosperm Phylogeny Group classification for the orders and families of flowering plants: APG III. Botanical Journal of the Linnean Society 161: 105-121.

Appel, O. \& I.A. Al-Shehbaz. 2003. Cruciferae. In: K. Kubitzki $\&$ C. Bayer (eds.). The Families and Genera of Vascular Plants, Vol. 5, pp. 75-174. Springer, New York.

ApPlequist, W.L. 2012. Report of the Nomenclature Committee for Vascular Plants: 64. Taxon 61(5): 1108-1117. 
Arakaki, M., C. Ostolaza, F. Cáceres \& J. Roque. 2006. Cactaceae endémicas del Perú. Revista Peruana de Biología 13(2): 193-219.

BACKEBERG, C. 1958. Die Cactaceae: Handbuch der Kakteenkunde. Band 1. Pereskioideae und Opuntioideae. VEB Gustav Fischer Verlag Jena 1-638.

BACKeBerg, C. 1959. Die Cactaceae: Handbuch der Kakteenkunde. Band 3. Cereoideae (Austrocactinae). VEB Gustav Fischer Verlag Jena 1361-1926.

Baeza, C.M., T.F. Stuessy \& C. Marticorena. 2002. Notes on the Poaceae of the Robinson Crusoe (Juan Fernández) Islands, Chile. Brittonia 54(3): 154-163.

Baeza, C.M., P. Novoa, E. Ruiz \& M.A. Negritto. 2009. El cariotipo fundamental en Traubia modesta (Phil.) Ravenna (Amaryllidaceae). Gayana Botánica 66(2): 297-300.

Baldwin, B.G., B.L. Wessa \& J.L. Panero. 2002. Nuclear rDNA Evidence for Major Lineages of Helenioid Heliantheae (Compositae). Systematic Botany 27(1): 161-198.

Bárcenas, R.T., C. Yesson \& J.A. Hawkins. 2011. Molecular systematics of the Cactaceae. Cladistics 27: 1-20.

Barthlott, W. \& D.R. Hunt. 1993. Cactaceae. In: K. Kubitzki, J.G. Rohwer \& V. Bittrich (eds.). The Families and Genera of Vascular Plants, Vol. 2, pp. 161-197. Springer, New York.

Bayer, E. 1998. Alstroemeriaceae. In: K. Kubitzki (ed.). The Families and Genera of Vascular Plants, Vol. 3, pp. 79-83. Springer, New York.

Benoit, I.L. (ed.). 1989. Libro rojo de la Flora terrestre de Chile. CONAF, Santiago, Chile, 157 pp.

Bernardello, G., L. StiefKens \& M.L. Las Peñas. 2008. Karyotype studies in Grabowskia and Phrodus (Solanaceae). Plant Systematics and Evolution 275: 265-269.

Bittrich, V. 1993. Caryophyllaceae. In: K. Kubitzki, G. Rohwer $\&$ V. Bittrich (eds.). The Families and Genera of Vascular Plants, Vol. 2, pp. 206-236. Springer, New York.

Blanco, S., M.L. Las Peñas, G. Bernardello \& L. Stiefkens. 2012. Mapeo de genes ribosómicos y heterocromatina en seis especies de Lycium de Sudamérica (Solanaceae). Boletin de la Sociedad Argentina de Botánica 47(3-4): 389-399.

Bonifacino, J.M. \& G. SAncho. 2004. Guynesomia (Asteraceae: Astereae), a New Genus from Central Chile. Taxon 53(3): 673-678.

BraKo, L. \& J.L. ZaruCCHI. 1993. Catalogue of the flowering plants and gymnosperms of Peru. Monographs in Systematic Botany Vol. 45. Missouri Botanical Garden Press, St. Louis. 1286 pp.

Britton, N.L. \& J.N. Rose. 1922. The Cactaceae. Descriptions and Illustrations of the Plants of the Cactus Family, Vol. III. Carnegie Institution, Washington D.C., 255 pp.

Bruneau, A., F. Forest, P.S. Herendeen, B.B. Klitgaard \& G.P. LewIS. 2001. Phylogenetic Relationships in the Caesalpinioideae (Leguminosae) as Inferred from Chloroplast trnL Intron Sequences. Systematic Botany 26(3): 487-514.

Bruneau, A., M. Mercure, G.P. Lewis \& P.S. Herendeen. 2008. Phylogenetic patterns and diversification in the caesalpinioid legumes. Botany 86: 697-718.

Buerki, S., J.C. Manning \& F. Forest. 2013. Spatio-temporal history of the disjunct family Tecophilaeaceae: a tale involving the colonization of three Mediterranean-type ecosystems. Annals of Botany 111: 361-373.

Buerki, S., F. Forest, P. Acevedo-Rodríguez, M.W. Callmander, J.A.A. Nylander, M. Harrington, I. SAnMartín, P. KüPfer \& N. Alvarez. 2009. Plastid and nuclear DNA markers reveal intricate relationships at subfamilial and tribal levels in the soapberry family (Sapindaceae). Molecular Phylogenetics and Evolution 51: 238-258.

Cabrera, A.L. 1948. Notas sobre la vegetación de la Puna Argentina. Anales de la Academia Nacional de Ciencias Exactas, Físicas y Naturales de Buenos Aires 12: 15-38.

Caddick, L.R., P.J. Rudall, P. Wilkin, T.A.J. Hedderson \& M.W. ChASE. 2002a. Phylogenetics of Dioscoreales based on combined analyses of morphological and molecular data. Botanical Journal of the Linnean Society 138: 123-144.

Caddick, L.R., P. Wilkin, P.J. Rudall, T.A.J. Hedderson \& M.W. Chase. 2002b. Yams Reclassified: A Recircumscription of Dioscoreaceae and Dioscoreales. Taxon 51: 103-114.

Cameron, K.M., M.W. Chase, W.R. Anderson \& H.G. Hills. 2001. Molecular systematics of Malpighiaceae: Evidence from plastid rbcL and matK sequences. American Journal of Botany 88(10): 1847-1862.

CARlquist, S. 1958. Anatomy and systematic position of Centaurodendron and Yunquea (Compositae). Brittonia 10: 78-93.

Carlquist, S. 1967. Anatomy and systematics of Dendroseris (sensu lato). Brittonia 19: 99-121.

Chacón, J., M. Camargo de Assis, A.W. Meerow \& S.S. Renner. 2012. From East Gondwana to Central America: Historical biogeography of the Alstroemeriaceae. Journal of Biogeography 39: 1806-1818.

Charles, G. 1998. Copiapoa. The Cactus File Handbook, Vol. 4. Cirio Publishing, Service Ltd., Southampton. 80 pp.

Chase, M.W., D.E. Soltis, R.G. Olmstead, D. Morgan, D.H. Les, B.D. Mishler, M.R. Duvall, R.A. Price, H.G. Hills, Y.L. Qiu, K.A. Kron, J.H. Rettig, E. Conti, J.D. Palmer, J.R. Manhart, K.J. Sytsma H.J. Michaels, W.J. Kress, K.G. Karol, W.D. Clark, M. Hedren, B.S. Gaut, R.K. JANSEN, K.J. Kim, C.F. Wimpee, J.F. SMith, G.R. Furnier, S.H. Strauss, Q.Y. Xiang, G.M. Plunkett, P.S. Soltis, S.M. Swensen, S.E. Williams, P.A. Gadek, C.J. Quinn, L.E. Eguiarte, E. Golenberg, G.H. Learn, S.W. Graham, S.C.H. Barrett, S. Dayanandan \& V.A. Albert. 1993. Phylogenetics of Seed Plants: An analysis of nucleotide sequences from the plastid gene rbcL. Annals of the Missouri Botanical Garden 80: 528-580.

Chase, M.W., J.L. Reveal \& M.F. Fay. 2009. A subfamilial classification for the expanded asparagalean families Amaryllidaceae, Asparagaceae and Xanthorrhoeaceae. Botanical Journal of the Linnean Society 161: 132-136.

Cheng-Yin, W. \& K. Kubitzki. 1993. Lardizabalaceae. In: K. Kubitzki, G. Rohwer \& V. Bittrich (eds.). The Families and Genera of Vascular Plants, Vol. 2, pp. 361-365. Springer, New York.

Christenhusz, M.J.M. 2012. An overview of Lardizabalaceae. Curtis's Botanical Magazine 29(3): 235-276.

Christenhusz, M.J.M. \& M.W. Chase. 2014. Trends and concepts in fern classification. Annals of Botany 113: 571-594.

Christenhusz, M.J.M., X.C. Zhang \& H. Schneider. 2011. A linear sequence of extant families and genera of lycophytes and ferns. Phytotaxa 19: 7-54. 
Cisternas, M.A., L. Araneda, N. García \& C.M. Baeza. 2010. Karyotypic studies in the Chilean genus Placea (Amaryllidaceae). Gayana Botanica 67(2): 198-205.

Conran, J.G. \& H.T. Clifford. 1998. Philesiaceae. In: K. Kubitzki (ed.). The Families and Genera of Vascular Plants, Vol. 3, pp. 409-411. Springer, 478 pp.

Cosner, M.E., R.K. Jansen \& T.G. LAmmers. 1994. Phylogenetic relationships in the Campanulales based on rbcL sequences. Plant Systematics and Evolution 190: 79-95.

Cosner, M.E., L.A. Raubeson \& R.K. JAnsen. 2004. Chloroplast DNA rearrangements in Campanulaceae: Phylogenetic utility of highly rearranged genomes. BMC Evolutionary Biology 4: 27.

Crawford, D.J., S. Brauner, M.B. Cosner \& T.F. Stuessy. 1993. Use of RAPD markers to document the origin of the intergeneric hybrid xMargyracaena skottsbergii (Rosaceae) on the Juan Fernandez Islands. American Journal of Botany 80(1): 89-92.

Crisci, J.V. 1974a. Marticorenia: A New Genus of Mutisieae (Compositae). Journal of the Arnold Arboretum 55: 38-45.

CRISCI, J.V. 1974b. Revision of the Genus Moscharia (Compositae: Mutisieae) and a reinterpretation of its Inflorescence. Contributions from the Gray Herbarium of Harvard University 205: 163-173.

Danton, P., C. Perrier \& G. Martínez-Reyes. 2006. Nouveau catalogue de la flore vasculaire de l'archipel Juan Fernández (Chili). Acta Botanica Gallica 153(4): 399-587.

DAvis, C.C. \& W.R. Anderson. 2010. A complete generic phylogeny of Malpighiaceae inferred from nucleotide sequence data and morphology. American Journal of Botany 97(12): 2031-2048.

Davis, C.C., W.R. Anderson \& M.J. Donoghue. 2001. Phylogeny of Malpighiaceae: Evidence from chloroplast ndhF and trnL-F nucleotide sequences. American Journal of Botany 88(10): 1830-1846.

Ditrrich, M. 1977. Cynareae - systematic review. In: V.H. Heywood, J.B. Harborne \& B.L. Turner (eds). The biology and chemistry of the Compositae, Vol. 2. pp. 999-1015. Academic Press, London.

Donald, J.D. \& G.D. Rowley. 1966. Reunion of the genus Neoporteria. Cactus and Succulent Journal of Great Britain 28(3-4): 54-63.

Doweld, A.B. 2001a. On the phylogeny and systematics of the genus Copiapoa Britton et Rose (Copiapoeae Doweld Cactaceae Durande). Sukkulenty 4(1-2): 46-56.

Doweld, A.B. 2001b. Carpology and phermatology of Gomortega (Gomortegaceae): Systematic and evolutionary implications. Acta Botanica Malacitana 26: 19-37.

Dransfield, J. \& N.W. Uhl. 1998. Palmae. In: K. Kubitzki (ed.). The Families and Genera of Vascular Plants, Vol. 4, pp. 306-388. Springer, New York.

Duarte, M., P.C. Guerrero, G. Carvallo \& R.O. Bustamante. 2014. Conservation network design for endemic cacti under taxonomic uncertainty. Biological Conservation 176: 236-242.

Edwards, J.L., M.A. LANE \& E.S. NielSEN. 2000. Interoperability of Biodiversity Databases: Biodiversity Information on Every Desktop. Science 289: 2312-2314.

Ehrhart, C. 2001. Zephyra compacta (Tecophilaeaceae) - eine neue Art aus Chile. Sendtnera 7: 47-52.
Escobar, I. 2012. Sistemática de la tribu Gilliesieae Lindl. (Alliaceae), sobre la base de evidencias morfoanatómicas, citológicas y moleculares. Tesis doctoral, Universidad de Concepción, 217 pp.

Escobar, I., P. Novoa, E. Ruiz, M. Negritto \& C. Baeza. 2010. Nuevo hallazgo de Miersia cornuta Phil. (GilliesieaeAlliaceae). Gayana Botánica 67(1): 130-134.

Essi, L., H.M. Longhi-Wagner \& T.T. de SouzA-Chies. 2008. Phylogenetic analysis of the Briza complex (Poaceae). Molecular Phylogenetics and Evolution 47(3): 1018-1029.

Essi, L., H.M. Longhi-Wagner \& T.T. de Souza-Chies. 2011. New Combinations within the Briza Complex (Poaceae, Pooideae, Poeae). Novon 21(3): 326-330.

Eyzaguirre, M.T. \& R. García de la Huerta. 2002. Tecophilaea cyanocrocus Leyb. (Tecophilaeaceae) redescubierta en su hábitat natural. Gayana Botanica 59(2): 73-77.

FAY, M.F. \& M. CHASE. 1996. Resurrection of Themidaceae for the Brodiaea alliance, and recircumscription of Alliaceae, Amaryllidaceae and Agapanthoideae. Taxon 45(3): 441451.

FAY, M.F. \& T. HALl. 2007. Gethyum atropurpureum. Curtis's Botanical Magazine 24(2): 121-126.

Freire, S.E., J.V. Crisci \& L. Katinas. 1993. A cladistic analysis of Nassauvia Comm. ex Juss. (Asteraceae, Mutisieae) and related genera. Botanical Journal of the Linnean Society 112: 293-309.

FrIES, R.E. 1905. Zur Kenntnis Der Alpinen Flora Im Nördlichen Argentinien. Nova Acta Regiae Societatis Scientiarum Upsaliensis, Ser. 4, 1(1): 1-205.

García, N., A.W. Meerow, D.E. Soltis \& P.S. Soltis. 2014. Testing Deep Reticulate Evolution in Amaryllidaceae Tribe Hippeastreae (Asparagales) with ITS and Chloroplast Sequence Data. Systematic Botany 39(1): 75-89.

Givnish, T.J., M.H.J. Barfuss, B. Van Ee, R. Riina, K. Schulte, R. Horres, P.A. Gonsiska, R.S. Jabaily, D.M. Crayn, J.A.C. Smith, K. Winter, G.K. Brown, T.M. Evans, B.K. Holst, H. Luther, W. Till, G. Zizka, P.E. Berry \& K.J. Sytsma. 2011. Phylogeny, adaptive radiation, and historical biogeography in Bromeliaceae: Insights from an eight-locus plastid phylogeny. American Journal of Botany 98(5): 872-895.

González, F. \& P. Rudall. 2001. The questionable affinities of Lactoris: evidence from branching pattern, inflorescence morphology, and stipule development. American Journal of Botany 88(12): 2143-2150.

Grau, J. 1997. Huidobria, eine isolierte Gattung der Loasaceae aus Chile. Sendtnera 4: 77-93.

Griffith, M.P. \& J.M. Porter. 2009. Phylogeny of Opuntioideae (Cactaceae). International Journal of Plant Science 170: 107-116.

Groppo, M., J.A. Kallunki, J. Rubens-Pirani \& A. Antonelli. 2012. Chilean Pitavia more closely related to Oceania and Old World Rutaceae than to Neotropical groups: evidence from two cpDNA non-coding regions, with a new subfamilial classification of the family. PhytoKeys 19: 9-29.

Guerrero, P.C., A.C. Sandoval \& P. León-Lobos. 2007. The effect of chilling on seed germination of Placea species (Asparagales: Amaryllidaceae), an endemic genus to central Chile. Gayana Botánica 64(1): 40-45. 
Guerrero, P.C., M.T.K. Arroyo, R.O. Bustamante, M. Duarte, T.K. Hagemann \& H.E. Walter. 2011. Phylogenetics and predictive distribution modeling provide insights into the geographic divergence of Eriosyce subgen. Neoporteria (Cactaceae). Plant Systematics and Evolution 297: 113128.

Gustafsson, M.H.G., A. Backlund \& B. Bremer. 1996. Phylogeny of the Asterales sensu lato based on rbcL sequences with particular reference to the Goodeniaceae. Plant Systematics and Evolution 199: 217-242.

Harold, A.S. \& R.D. Moor. 1994. Areas of endemism: Definition and recognition criteria. Systematic Biology 43(2): 261266.

Hernández-Hernández, T., H.M. Hernández, J.A. De-Nova, R. Puente, L.E. Equiarte \& S. Magallón. 2011. Phylogenetic relationships and evolution of Growth form in Cactaceae (Caryophyllales, Eudicotyledoneae). American Journal of Botany 98(1): 44-61.

HinD, D.J.N. 2007. Tribe Mutisieae. In: J.W. Kadereit \& C. Jeffrey (eds.). The Families and Genera of Vascular Plants, Vol. 8, pp. 90-122. Springer, New York.

Hoffmann, A. \& H. Walter. 2004. Cactáceas en la Flora Silvestre

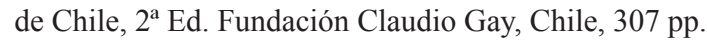

Horres, R., G. Zizka, G. Kahl \& K. Weising. 2000. Molecular Phylogenetics of Bromeliaceae: Evidence from trnL (UAA) Intron Sequences of the Chloroplast Genome. Plant biology 2(3): 306-315.

Huber, H. 1998. Dioscoreaceae. In: K. Kubitzki (ed.). The Families and Genera of Vascular Plants, Vol. 3, pp. 216235. Springer, New York.

Hufford, L., M.M. McMahon, A.M. Sherwood, G. Reeves \& M.W. Chase. 2003. The major clades of Loasaceae: Phylogenetic analysis using the plastid matK and trnL-trnF regions. American Journal of Botany 90(8): 1215-1228.

Hufford, L., M.M. McMahon, R. O’Quinn \& M.E. Poston. 2005. A phylogenetic analysis of Loasaceae subfamily Loasoideae based on plastid DNA sequences. International Journal of Plant Science 166(2): 289-300.

Hunt, D.R., N.P. Taylor \& G. Charles. 2006. The new cactus lexicon. DH Books, England. 899 pp.

Hunt, D.R. \& N.P. TAYLOR (eds.). 1986. The genera of Cactaceae: Towards a new consensus. Preliminary findings of an ad hoc Working Party under the auspices of the IOS. Bradleya 4: 65-78.

JABAILY, R.S. \& K.J. SytSma. 2010. Phylogenetics of Puya (Bromeliaceae): Placement, Major Lineages, and Evolution of Chilean Species. American Journal of Botany 97(2): 337-356.

JARA-Arancio, P. 2010. Reconstrucción filogenética del género endémico Leucocoryne (Alliaceae) y su correspondencia biogeográfica con la aridización de la zona mediterránea árida y semiárida de Chile. Tesis doctoral en Ciencias, Universidad de Chile, Santiago, Chile. 148 pp.

Johnston, I.M. 1927. Studies in the Boraginaceae VI. A revision of the South American Boraginoideae. Contributions from the Gray Herbarium of Harvard University 78: 1-118.

Jørgensen, P.M. \& S. León-YÁnez (eds.). 1999. Catalogue of the vascular plants of Ecuador. Monograph of Systematic Botany from the Missouri Botanical Garden 75: i-viii, 1-1182.
Kalkman, C. 2004. Rosaceae. In: K. Kubitzki (ed.). The Families and Genera of Vascular Plants, Vol. 6, pp. 343-386. Springer, New York.

Karaman-Castro, V. \& L.E. Urbatsch. 2009. Phylogeny of Hinterhubera Group and Related Genera (Hinterhuberinae: Astereae) based on the nrDNA ITS and ETS Sequences. Systematic Botany 34(4): 805-817.

Katinas, L. \& J.V. CRisci. 2000. Cladistic and Biogeographic Analyses of the Genera Moscharia and Polyachyrus (Asteraceae, Mutisieae). Systematic Botany 25(1): 33-46.

Katinas, L., J. Pruski, G. Sancho \& M.C. Tellería. 2008a. The Subfamily Mutisioideae (Asteraceae). Botanical Review 74: 469-716.

Katinas, L., J.V. Crisci, R. Schmidt-Jabaily, C. Williams, J. Walker, B. Drew, J.M. Bonifacino \& K.J. Sytsma. 2008 b. Evolution of secondary heads in Nassauviinae (Asteraceae, Mutisieae). American Journal of Botany 95(2): 229-240.

Kattermann, F. 1994. Eriosyce (Cactaceae). The genus revised and amplified. Succulent Plant Research 1: 1-176.

Kiesling, R., J. Larroca, L. Faúndez, D. Metzing \& S. Albesiano. 2008. Cactaceae. En: F.O. Zuloaga, O. Morrone \& M.J. Belgrano (eds.). Catálogo de las Plantas Vasculares del Cono Sur de América (Argentina, Sur de Brasil, Chile, Paraguay y Uruguay), Vol. 2: Dicotyledonae: AcanthaceaeFabaceae (Abarema-Schizolobium). Monographs in Systematic Botany from the Missouri Botanical Garden 107: 1715-1830.

Kilian, N., B. Gemeinholzer \& H.W. Lack. 2009. Cichorieae. In: V.A. Funk, A. Susanna, T.F. Stuessey \& R.J. Bayer (eds.). Systematics, Evolution, and Biogeography of Compositae, pp. 343-383. International Association for Plant Taxonomy, Vienna.

Kim, S.C., D.J. Crawford \& R.K. Jansen. 1996. Phylogenetic Relationships among the Genera of the Subtribe Sonchinae (Asteraceae): Evidence from ITS Sequences. Systematic Botany 21(3): 417-432.

Kim, S.C., D.J. Crawford, R.K. Jansen \& A. SAntos-Guerra. 1999. The use of a non-coding region of chloroplast DNA in phylogenetic studies of the subtribe Sonchinae (Asteraceae: Lactuceae). Plant Systematics and Evolution 215: 85-99.

Kim, S.C., L. Chunghee, J.A. Mejias. 2007. Phylogenetic analysis of chloroplast DNA matK gene and ITS of nrDNA sequences reveals polyphyly of the genus Sonchus and new relationships among the subtribe Sonchinae (Asteraceae: Cichorieae). Molecular Phylogenetics and Evolution 44: 578-597.

Kubitzki, K. 1993a. Gomortegaceae. In: K. Kubitzki, G. Rohwer $\&$ V. Bittrich (eds.). The Families and Genera of Vascular Plants, Vol. 2, pp. 318-320. Springer, New York.

Kubitzki, K. 1993b. Plumbaginaceae. In: K. Kubitzki, G. Rohwer \& V. Bittrich (eds.). The Families and Genera of Vascular Plants, Vol. 2, pp. 523-530. Springer, New York.

KubitzKi, K. 1993c. Lactoridaceae. In: K. Kubitzki, G. Rohwer \& V. Bittrich (eds.). The Families and Genera of Vascular Plants, Vol. 2, pp. 359-361. Springer, New York.

Kubitzki, K., J.A. Kallunki, M. Duretto \& P.G. Wilson. 2011. Rutaceae. In: K. Kubitzki (ed.). The Families and Genera of Vascular Plants, Vol. 10, pp. 276-356. Springer, New York. 
KuIJ, J. 1985. Morphology, Biology, and Systematic Relationships of Desmaria (Loranthaceae). Plant Systematics and Evolution 151: 121-130.

Kulu, K.K.M., S.E.C. Sierra, S.G.A. Draisma, R. SAmuel \& P.C. VAN Welzen. 2007. Molecular phylogeny of Macaranga, Mallotus, and related genera (Euphorbiaceae s.s.): insights from plastid and nuclear DNA sequence data. American Journal of Botany 94(10): 1726-1743.

Kuo, L.Y., F.W. Li, W.L. Chiou \& C.N. WAng. 2011. First insights into fern matK phylogeny. Molecular Phylogenetics and Evolution 59(3): 556-566.

Landrum, L. 1988. The Myrtle family (Myrtaceae) in Chile. Proceedings of the California Academy of Sciences 45(12): 277-317.

Lammers, T.G. 2007. Campanulaceae. In: J.W. Kadereit \& C. Jeffrey (eds.). The Families and Genera of Vascular Plants, Vol. 8, pp. 26-56. Springer, New York.

Lee, C., S.C. Kim, K. Lundy \& A. Santos-Guerra. 2005. Chloroplast DNA Phylogeny of the Woody Sonchus Alliance (Asteraceae: Sonchinae) in the Macaronesian Islands. American Journal of Botany 92: 2072-2085.

LEFor, M.W. 1975. A taxonomic revision of the Vivianiaceae. University of Connecticut, Occasional Papers, Biological Science Series 2: 225-255.

Lehtonen, S. 2011. Towards Resolving the Complete Fern Tree of Life. PLoS ONE 6(10): e24851.

Letelier, L., F.A. Squeo, G. Arancio, A. Marticorena, M. MuñozSchick, M.T.K. Arroyo, P. León-Lobos, S. Montecinos \& J.R. GutiérRez. 2008. Diversidad vegetal de la Región de Atacama, Chile. En: F.A. Squeo, G. Arancio \& J.R. Gutiérrez (eds.). Libro Rojo de la flora nativa y de los sitios prioritarios para su Conservación: Región de Atacama, pp. 123-135. Ediciones Universidad de La Serena, La Serena, Chile.

LeVIN, R.A. \& J.S. Miller. 2005. Relationships within tribe Lycieae (Solanaceae): Paraphyly of Lycium and multiple origins of gender dimorphism. American Journal of Botany 92(12): 2044-2053.

Levin, R.A., J. Blanton \& J.S. Miller. 2009a. Phylogenetic utility of nuclear nitrate reductase: A multi-locus comparison of nuclear and chloroplast sequence data for inference of relationships among American Lycieae (Solanaceae). Molecular Phylogenetics and Evolution 50: 608-617.

Levin, R.A., A. Whelan \& J.S. Miller. 2009b. The utility of nuclear conserved ortholog set II (COSII) genomic regions for species-level phylogenetic inference in Lycium (Solanaceae). Molecular Phylogenetics and Evolution 53: 881-890.

Levin, R.A., G. Bernardello, C. Whiting \& J.S. Miller. 2011. A new generic circumscription in tribe Lycieae (Solanaceae). Taxon 60(3): 681-690.

Lia, V.V., V.A. Confalonieri, C.I. Comas \& J.H. Hunziker. 2001. Molecular Phylogeny of Larrea and Its Allies (Zygophyllaceae): Reticulate Evolution and the Probable Time of Creosote Bush Arrival to North America. Molecular Phylogenetics and Evolution 21(2): 309-320.

LindER, H.P. 2001. On areas of endemism, with an example from the African Restionaceae. Systematic Biology 50(6): 892912.

LindER, H.P. 2007. Melianthaceae. In: K. Kubitzki (ed.). The
Families and Genera of Vascular Plants, Vol. 9, pp. 250259. Springer, New York.

Liu, M., G.M. Plunkett, B.E. Van WyK, P.M. Tilney \& P.P. Lowry II. 2012. The phylogenetic significance of the carpophore in Apiaceae. Annals of Botany 110: 1531-1543.

Lledó, M.D., M. Erben \& M.B. Crespo. 2003. Myriolepis, a new genus segregated from Limonium (Plumbaginaceae). Taxon 52: 67-73.

Lledó, M.D., M.B. Crespo, M.F. Fay \& M.W. Chase. 2005. Molecular phylogenetics of Limonium and related genera (Plumbaginaceae): Biogeographical and systematic implications. American Journal of Botany 92(7): 11891198.

Luebert, F. \& P. BeCERRA. 1998. Representatividad vegetacional del Sistema Nacional de Áreas Silvestres Protegidas del Estado (SNASPE) en Chile. Ambiente y Desarrollo 14(2): 62-69.

Luebert, F., J. Wen \& M.O. Dillon. 2009. Systematic placement and biogeographical relationships of the monotypic genera Gypothamnium and Oxyphyllum (Asteraceae: Mutisioideae) from the Atacama Desert. Botanical Journal of the Linnean Society 159: 32-51.

Lundberg, J. 2001. Phylogenetic studies in the Euasterids II with particular reference to Asterales and Escalloniaceae. Comprehensive Summaries of Uppsala Dissertations from the Faculty of Science and Technology Acta Universitatis Upsaliensis. 676. 38 pp.

Lundberg, J. \& K. Bremer. 2003. A phylogenetic study of the Order Asterales using one morphological and three molecular data sets. International Journal of Plant Science 164(4): 553-578.

Lüthy, J. 1994. Appendix II. Eriosyce laui J. Lüthy sp. nov. In: F. Kattermann, Eriosyce (Cactaceae). The genus revised and amplified. Succulent Plant Research 1: 120-124.

Manzanilla, V. \& A. Bruneau. 2012. Phylogeny reconstruction in the Caesalpinieae grade (Leguminosae) based on duplicated copies of the sucrose synthase gene and plastid markers. Molecular Phylogenetics and Evolution 65: 149162.

Maraner, F., R. Samuel, T.F. Stuessy, D.J. Crawford, J.V. Crisci, A. Pandey \& M.E. Mort. 2012. Molecular phylogeny of Nassauvia (Asteraceae, Mutisieae) based on nrDNA ITS sequences. Plant Systematics \& Evolution 298: 399-408.

Margules, C. \& R. Pressey. 2000. Systematic conservation planning. Nature 405: 243-253.

Marticorena, C. 1990. Contribución a la estadística de la Flora vascular de Chile. Gayana Botanica 47(3-4): 85-113.

Marticorena, C. 1995. Historia de la exploración botánica de Chile. In: C. Marticorena \& R. Rodríguez (eds.), Flora de Chile, Vol. 1, pp. 1-62. Ediciones Universidad de Concepción, Concepción, Chile.

Marticorena, C. \& M. QuezadA. 1985. Catálogo de la flora vascular de Chile. Gayana, Botánica 42(1-2): 1-157.

Martínez-Crovetto, R. 1967. Catálogo preliminar de las Caryophyllaceae de la Argentina y del Uruguay. Bonplandia 2(14): 187-264.

Maunder, M., R.S. Cowan, P. Stranc \& M.F. Fay. 2001. The genetic status and conservation management of two cultivated bulb species extinct in the wild: Tecophilaea cyanocrocus (Chile) and Tulipa sprengeri (Turkey). 
Conservation Genetics 2: 193-201.

Mayer, V., M. Möller, M. Perret \& A. Weber. 2003. Phylogenetic position and generic differentiation of Epithemateae (Gesneriaceae) Inferred from plastid DNA sequence data. American Journal of Botany 90(2): 321-329.

Medan, M. \& C. Schirarend. 2004. Rhamnaceae. In: K. Kubitzki (ed.). The Families and Genera of Vascular Plants, Vol. 6, pp. 320-338. Springer, New York.

Meerow, A.W. \& D.A. Snijman. 1998. Amaryllidaceae. In: K. Kubitzki (ed.). The Families and Genera of Vascular Plants, Vol. 3, pp. 83-110. Springer, New York.

Mejías, J.A. \& S.C. KIM. 2012. Taxonomic treatment of Cichorieae (Asteraceae) endemic to the Juan Fernández and Desventuradas Islands (SE Pacific). Annales Botanici Fennici 49: 171-178.

Miller, J.S., A. Kamath \& R.A. Levin. 2009. Do Multiple Tortoises Equal a Hare? The Utility of Nine Noncoding Plastid Regions for Species-Level Phylogenetics in Tribe Lycieae (Solanaceae). Systematic Botany 34(4): 796-804.

Ministerio Secretaría General de la Presidencia(MinSEGPRES). 2005. DeCreto Supremo N ${ }^{\circ} 75 / 2005$. Aprueba Reglamento para la Clasificación de Especies Silvestres. Diario oficial de la República de Chile. Publicado el 11 de mayo de 2005.

Ministerio del Medio Ambiente (MMA). 2012. Decreto Supremo $N^{\circ}$ 29/2012. Aprueba Reglamento para la Clasificación de Especies Silvestres según estado de conservación. Diario oficial de la República de Chile. Publicado el 27 de abril de 2012.

Ministerio del Medio Ambiente (MMA). 2012. Decreto Supremo $N^{\circ} 33 / 2012$. Aprueba y oficializa clasificación de especies según su estado de conservación, quinto proceso. Diario oficial de la república de Chile. Publicado el 27 de febrero de 2012.

Mittermeier, R.A., P.R. Gil, M. Hoffmann, J. Pilgrim, T. Brooks, C.G. Mittermeier, J. Lamoreux \& G.A.B. da Fonseca. 2004. Hotspots revisited: Earth's biologically richest and most endangered terrestrial ecoregions. Conservation International, Washington DC. 392 pp.

Möller, M., M. Pfosser, C.G. Jang, V. Mayer, A. Clark, M.L. Hollingsworth, M.H.J. Barfuss, Y.Z. Wang, M. Kiehn \& A. Weber. 2009. A preliminary phylogeny of the 'didymocarpoid Gesneriaceae' based on three molecular data sets: Incongruence with available tribal classifications. American Journal of Botany 96(5): 989-1010.

Moody, M.L., L. Hufford, D.E. Soltis \& P.S. Soltis. 2001. Phylogenetic relationships of Loasaceae subfamily Gronovioideae inferred from matK and ITS sequence data. American Journal of Botany 88(2): 326-336.

Moreira-Muñoz, A. 2011. Plant Geography of Chile. Plant and Vegetation Series, Vol. 5, Springer, New York. 343 pp.

Moreira-Muñoz, A. \& M. MuÑoz-Schick. 2007. Classification, diversity, and distribution of Chilean Asteraceae: implications for biogeography and conservation. Diversity and Distributions 13: 818-828.

Moreira-Muñoz, A., V. Morales \& M. Muñoz-Schick. 2012. Actualización sistemática y distribución geográfica de Mutisioideae (Asteraceae) de Chile. Gayana Botánica 69(1): 9-29.

Morgan, D.R. \& D.E. Soltis. 1993. Phylogenetic relationships among members of Saxifragaceae sensu lato based on
rbcL sequence data. Annals of the Missouri Botanical Garden 80: 631-660.

Mottram, R. 2001. Rimacactus, a new genus of Cactaceae. Bradleya 19: 75-82.

Muñoz-SchicK, M. 2000. Consideraciones sobre géneros endémicos de monocotiledóneas en Chile. Noticiario Mensual del Museo Nacional de Historia Natural 343: 1627.

Muñoz-Schick, M. \& A. Moreira-Muñoz. 2000. Los géneros endémicos de Monocotiledóneas de Chile continental. URL: $\quad$ http://www.chlorischile.cl/Monocotiledoneas/ Principalbot.htm Visitado el 28 de enero de 2015.

Muñoz-Schick, M. \& V. Morales. 2013. Complemento y Correcciones al "Catálogo de Plantas Vasculares del Cono Sur", para la Flora de Chile. Boletín del Museo Nacional de Historia Natural 62: 167-201.

Murillo-Aldana, J. \& E. Ruiz. 2011. Revalidación de Nothomyrcia (Myrtaceae), un género endémico del Archipiélago de Juan Fernández. Gayana Botánica 68(2): 129-134.

Murrilo-Aldana, J., E. Ruiz-P., L.R. Landrum, T.F. Stuessy \& M.H.J. BARfuss. 2012. Phylogenetic relationships in Myrceugenia (Myrtaceae) based on plastid and nuclear DNA sequences. Molecular Phylogenetics and Evolution 62: 764-776.

Murillo-Aldana, J., T.F. Stuessy \& E. Ruiz. 2013. Phylogenetic relationships among Myrceugenia, Blepharocalyx, and Luma (Myrtaceae) based on paired-sites models and the secondary structures of ITS and ETS sequences. Plant Systematics and Evolution 299: 713-729.

Myers, N., R.A. Mittermeier, C.G. Mittermeier, G.A.B. DA Fonseca \& J. Kent. 2000. Biodiversity hotspots for conservation priorities. Nature 403: 853-858.

Naumann, J., K. Salomo, J.P. Der, E.K. Wafula, J.F. Bolin, E. Maass, L. Frenzke, M.S. Samain, C. Neinhuis, C.W. De Pamphilis \& S. Wanke. 2013. Single-Copy Nuclear Genes Place Haustorial Hydnoraceae within Piperales and Reveal a Cretaceous Origin of Multiple Parasitic Angiosperm Lineages. PLoS ONE 8(11): e79204.

Neinhuis, C., S. Wanke, K.W. Hilu, K. Müller \& T. Borsch. 2005. Phylogeny of Aristolochiaceae based on parsimony, likelihood, and Bayesian analyses of trnL-trnF sequences. Plant Systematics and Evolution 250: 7-26.

Nesom, G. \& H. Robinson. 2007. Tribe Astereae, pp. 284-342. In: J.W. Kadereit \& C. Jeffrey (eds.). The Families and Genera of Vascular Plants, Vol. 8, Springer, 509 pp.

Nickrent, D.L., V. Malécot, R. Vidal-Russell \& J.P. Der. 2010. A revised classification of Santalales. Taxon 59(2): 538558.

Nicolas, A.N. \& G.M. Plunkett. 2009. The demise of subfamily Hydrocotyloideae (Apiaceae) and the re-alignment of its genera across the entire order Apiales. Molecular Phylogenetics and Evolution 53: 134-151.

Nores, M.J., B.B. Simpson, P. Hick, A.M. Anton \& R.H. Fortunato. 2012. The phylogenetic relationships of four monospecific caesalpinioids (Leguminosae) endemic to southern South America. Taxon 61(4): 790-802.

Nowicke, J. 1968. Palynotaxonomic Study of the Phytolaccaceae. Annals of the Missouri Botanical Garden 55(3): 294-364.

Nyffeler, R. \& U. Eggli. 1997. Comparative stem anatomy and systematics of Eriosyce sensu lato (Cactaceae). Annals of 
Botany 80: 767-786.

Nyffeler, R. \& U. EgGli. 2010. A Farewell to dated ideas and concepts: molecular phylogenetics and a revised suprageneric classification of the family Cactaceae. Schumannia 6: 109-149.

Nyffeler, R., U. Eggli \& B.E. Leuenberger. 1997. Noteworthy idioblastic sclereids in the stems of Eulychnia (Cactaceae). American Journal of Botany 84: 1192-1197.

Olmstead, R.G. \& J.D. Palmer. 1992. A Chloroplast DNA Phylogeny of the Solanaceae: Subfamilial Relationships and Character Evolution. Annals of the Missouri Botanical Garden 79(2): 346-360.

Olmstead, R.G., L. Bohs, H. Abdel Migid, E. Santiago-Valentin, V.F. García \& S.M. Collier. 2008. A Molecular Phylogeny of the Solanaceae. Taxon 57(4): 1159-1181.

Ostolaza, C. 2011. 101 cactus del Perú. Ministerio del Ambiente, Lima. 253 pp.

Palazzesi, L., M. Gottschling, V. Barreda \& M. Weigend. 2012. First Miocene fossils of Vivianiaceae shed new light on phylogeny, divergence times, and historical biogeography of Geraniales. Biological Journal of the Linnean Society 107: 67-85.

Palma-Rojas, C., P. Jara-Seguel \& E. von Brand. 2007. Karyological studies in Chilean species of Bomarea and Leontochir (Alstroemeriaceae). New Zealand Journal of Botany 45(2): 299-303.

Panero, J.L. 2007. Tribe Heliantheae Cass. (1819). In: J.W. Kadereit \& C. Jeffrey (eds.). The Families and Genera of Vascular Plants, Vol. 8, pp. 440-477. Springer, New York.

Panero, J.L. \& V.A. Funk. 2007. New Infrafamilial Taxa in Asteraceae. Phytologia 89(3): 356-360.

PAnero, J.L. \& V.A. Funk. 2008. The value of sampling anomalous taxa in phylogenetic studies: Major clades of the Asteraceae revealed. Molecular Phylogenetics and Evolution 47: 757782.

Pelser, P.B., B. Nordenstam, J.W. Kadereit \& L.E. Watson. 2007. An ITS Phylogeny of Tribe Senecioneae (Asteraceae) and a New Delimitation of Senecio L. Taxon 56(4): 1077-1104.

Pelser, P.B., E.J. Tepe, A.H. Kennedy \& L.E. Watson. 2010. The fate of Robinsonia (Asteraceae): sunk in Senecio, but still monophyletic?. Phytotaxa 5: 31-46.

Petersen, G., O. Seberg \& J.I. Davis. 2013. Phylogeny of the Liliales (Monocotyledons) with special emphasis on data partition congruence and RNA editing. Cladistics 29(3): 274-295.

Philipson, W.R. 1993. Monimiaceae. In: K. Kubitzki, G. Rohwer \& V. Bittrich (eds.). The Families and Genera of Vascular Plants, Vol. 2, pp. 426-437. Springer, New York.

Pitman, N.C.A. \& P.M. Jorgensen. 2002. Estimating the size of the world's threatened flora. Science 298: 989.

Pliscoff, P. \& T. Fuentes-Castillo. 2011. Representativeness of terrestrial ecosystems in Chile's protected area system. Environmental Conservation 38(3): 303-311.

Price, R.A. \& J.D. Palmer. 1993. Phylogenetic relationships of the Geraniaceae and Geraniales from rbcL sequence comparisons. Annals of the Missouri Botanical Garden 80: 661-671.

Qiu, Y.L., M.W. Chase, D.H. Les \& C.R. Parks. 1993. Molecular Phylogenetics of the Magnoliidae: Cladistic analyses of nucleotide sequences of the plastid gene rbcL. Annals of the Missouri Botanical Garden 80: 587-606.

Qiu, Y.L., J. Lee, F. Bernasconi-Quadroni, D.E. Soltis, P.S. Soltis, M. Zanis, E.A. Zimmer, Z. Chen, V. Savolainen \& M.W. CHASE. 2000. Phylogeny of Basal Angiosperms: Analyses of five genes from three genomes. International Journal of Plant Science 161: S3-S27.

RaHn, K. 1998. Alliaceae. In: K. Kubitzki (ed.). The Families and Genera of Vascular Plants, Vol. 3, pp. 70-78. Springer, New York.

Ravenna, P. 1981. Taxonomical notes on the Chilean Cruciferae. Nordic Journal of Botany 1: 140-142.

Ravenna, P. 1998. New or noteworthy Tecophilaeaceae. Phytologia 64: 288-289.

Ravenna, P. 2000. The family Gilliesiaceae. Onira 4(3): 11-14.

Ravenna, P. 2005. On the absence of the genus Gilliesia (Gilliesiaceae) in the Argentine Flora. Onira 9(15): 59.

Renner, S.S., J.S. StriJk, D. Strasberg \& C. Thébaud. 2010. Biogeography of the Monimiaceae (Laurales): a role for East Gondwana and long-distance dispersal, but not West Gondwana. Journal of Biogeography 37: 1227-1238.

RicArdi, M. 1958. Las especies chilenas del género Microphyes. Boletín de la Sociedad Argentina de Botánica 7(2): 120126.

Ricci, M. 2001. Evaluation of conservation status of Lactoris fernandeziana Philippi (Lactoridaceae) in Chile. Biodiversity and Conservation 10: 2129-2138.

Ritter, F. 1959. Chileorebutia Ritter gen. nov. ex Fric pro parte. Cactus, Organe de l'Association Francaise de Cactus et Plantes Grasses, Paris. 65: 191-194.

Ritter, F. 1980. Kakteen in Südamerika. Band 3: Chile. Spangerberg. pp. 857-1238.

Ritz, C.M., J. Reiker, G. Charles, P. Hoxey, D. Hunt, M. Lowry, W. Stuppy \& N. TAYLOR. 2012. Molecular phylogeny and character evolution in terete-stemmed Andean opuntias (Cactaceae-Opuntioideae). Molecular Phylogenetics and Evolution 65: 668-681.

Robinson, H. 1981. A Revision of the Tribal and Subtribal Limits of the Heliantheae (Asteraceae). Smithsonian Contributions to Botany, Vol. 51, Smithsonian Institution Press, 102 pp.

Rohwer, J.G. 1993. Phytolaccaceae. In: K. Kubitzki, G. Rohwer \& V. Bittrich (eds.). The Families and Genera of Vascular Plants, Vol. 2, pp. 506-515. Springer, New York.

Rodríguez, E.F., R. VÁsquez, R. Rojas, G. Calatayud, B. León \& J. CAmpos. 2006. Nuevas adiciones de angiospermas a la flora del Perú. Revista Peruana de Biología 13(1): 129138.

Rudall, P.J., R.M. Bateman, M.F. Fay \& A. Eastman. 2002. Floral anatomy and systematics of Alliaceae with particular reference to Gilliesia, a presumed insect mimic with strongly zygomorphic flowers. American Journal of Botany 89(12): 1867-1883.

Ruiz, E. 2003. Lardizabalaceae. In: C. Marticorena \& R. Rodríguez (eds.). Flora de Chile, Vol. 2(2), pp. 24-27. Universidad de Concepción, Concepción, Chile.

Ruiz, E., C. Marticorena, D. Crawford, T. Stuessy, F. González, R. Montoya, M. Silva \& J. Becerra. 2000. Morphological and ITS sequence divergence between taxa of Cuminia (Lamiaceae), an endemic genus of the Juan Fernandez Islands, Chile. Brittonia 52(4): 341-350.

Salariato, D.L., F.O. Zuloaga \& I.A. Al-Shehbaz. 2013. 
Molecular phylogeny of Menonvillea and recognition of the new genus Aimara (Brassicaceae: Cremolobeae). Taxon 62(6): 1220-1234.

Salas, P. \& L. Mansur. 2004. Gene Flow between Parents with Different Ploidy Levels in a Natural Population of Leucocoryne Lindley. Journal of the American Society for Horticultural Science 129(6): 833-835.

Saldivia, P., L. Faúndez, A. Marticorena \& J.L. Panero. 2014. Kieslingia chilensis (Asteraceae: Astereae), a new genus and species from northern Chile. Phytotaxa 177(5): 280290.

SAmpson, F.B. 1995. Pollen morphology of Lactoridaceae - a reexamination. Grana 34: 100-107.

Savolainen, V., M.F. Fay, D.C. Albach, A. Backlund, M. van der Bank, K.M. Cameron, S.A. Johnson, M.D. Lledó, J.C. Pintaud, M. Powell, M.C. Sheahan, D.E. Soltis, P.S. Soltis, P. Weston, W.M. Whitten, K.J. Wurdack \& M.W. Chase. 2000. Phylogeny of the eudicots: a nearly complete familial analysis based on $\mathrm{rbcL}$ gene sequences. Kew Bulletin 55: 257-309.

Scherson, R.A., A.A. Albornoz, A.S. Moreira-Muñoz \& R. UrbinA-CASANOVA. 2014. Endemicity and evolutionary value: a study of Chilean endemic vascular plant genera. Ecology and Evolution 4(6): 806-816.

Schneider, J., G. Winterfeld, M.H. Hoffmann \& M. Röser. 2011. Duthieeae, a new tribe of grasses (Poaceae) identified among the early diverging lineages of subfamily Pooideae: molecular phylogenetics, morphological delineation, cytogenetics and biogeography. Systematics and Biodiversity 9(1): 27-44.

Schuettpelz, E. \& K.M. Pryer. 2007. Fern phylogeny inferred from 400 leptosporangiate species and three plastid genes. Taxon 56(4): 1037-1050.

Schulte K., M.H.J. Barfuss \& G. Zizka. 2009. Phylogeny of Bromelioideae (Bromeliaceae) inferred from nuclear and plastid DNA loci reveals the evolution of the tank habit within the subfamily. Molecular Phylogenetics and Evolution 51: 327-339.

Schwarzer, C., F. Cáceres-Huamaní, A. Cano, M.I. La Torre \& M. WeIGEND. 2010. 400 years for long-distance dispersal and divergence in the northern Atacama Desert e Insights from the Huaynaputina pumice slopes of Moquegua, Peru. Journal of Arid Environments 74: 1540-1551.

Sede, S.M., S.I. Dürnhöfer, S. Morello \& F. Zapata. 2013. Phylogenetics of Escallonia (Escalloniaceae) based on plastid DNA sequence data. Botanical Journal of the Linnean Society 173: 442-451.

Sheahan, M.C. 2007. Zygophyllaceae. In: K. Kubitzki (ed.). The Families and Genera of Vascular Plants, Vol. 9, pp. 488500. Springer, New York.

Simpson, B.B. 1989. Pollination Biology and Taxonomy of Dinemandra and Dinemagonum (Malpighiaceae). Systematic Botany 14(3): 408-426.

Simpson, B.B., M.T.K. Arroyo, S. Sipe, M. Dias de Moraes \& J. McDill. 2009. Phylogeny and evolution of Perezia (Asteraceae: Mutisieae: Nassauviinae). Journal of Systematics and Evolution 47(5): 431-443.

Simpson M.G. \& P.J. Rudall. 1998. Tecophilaeaceae. In: K. Kubitzki (ed.). The Families and Genera of Vascular Plants, Vol. 3, pp. 429-436. Springer, New York.
Smith, L.B. \& W. Till. 1998. Bromeliaceae. In: K. Kubitzki (ed.). The Families and Genera of Vascular Plants, Vol. 4, pp. 74-99. Springer, New York.

Smith, A.R., K.M. Pryer, E. Schuettpelz, P. Korall, H. Schneider \& P.G. Wolf. 2006. A classification for extant ferns. Taxon 55(3): 705-731.

Smith-Ramírez, C., G. Arellano, E. Hagen, R. Vargas, J. Castillo \& A. Miranda. 2013. El rol de Turdus falcklandii (Aves: Passeriforme) como dispersor de plantas invasoras en el archipiélago de Juan Fernández. Revista Chilena de Historia Natural 86: 33-48.

Soltis, P.S. \& D.E. Soltis. 2009. The Role of Hybridization in Plant Speciation. Annual Review of Plant Biology 60: 561-588.

Soltis, D.E., P.S. Soltis, D.L. Nickrent, L.A. Johnson, W.J. Hahn, S.B. Hoot, J.A. Sweere, R.K. Kuzoff, K.A. Kron, M.W. Chase, S.M. Swensen, E.A. Zimmer, S.M. Chaw, L.J. Gillespie, W.J. Kress \& K.J. Sytsma. 1997. Angiosperm Phylogeny inferred from 18 S ribosomal DNA sequences. Annals of the Missouri Botanical Garden 84: 1-49.

Soltis, D.E., P.S. Soltis, M.W. Chase, M.E. Mort, D.C. Albach, M. Zanis, V. Savolainen, W.H. Hahn, S.B. Hoot, M.F. Fay, M. Axtell, S.M. Swensen, L.M. Prince, W.J. Kress, K.C. NiXon \& J.S. Farris. 2000. Angiosperm phylogeny inferred from 18S rDNA, rbcL, and atpB sequences. Botanical Journal of the Linnean Society 133: 381-461.

Squeo, F.A., G. Arancio, C. Marticorena, M. Muñoz \& J.R. GutiérRez. 2001. Diversidad vegetal de la IV Región de Coquimbo, Chile. En: F.A. Squeo, G. Arancio \& J.R. Gutiérrez (eds.). Libro Rojo de la flora nativa y de los sitios prioritarios para su Conservación: Región de Coquimbo, pp. 149-158. Ediciones Universidad de La Serena, La Serena, Chile.

Stuessy, T.F., D.J. Crawford, G.J. Anderson \& R.J. Jensen. 1998. Systematics, biogeography and conservation of Lactoridaceae. Perspectives in Plant Ecology, Evolution and Systematics 1/2: 267-290.

Susanna, A. \& N. Garcia-Jacas. 2007. Tribe Cardueae Cass. (1819), pp. 123-147. In: J.W. Kadereit \& C. Jeffrey (eds.). The Families and Genera of Vascular Plants Vol. 8, Springer, $635 \mathrm{pp}$.

Susanna, A., M. Galbany-Casals, K. Romaschenko, L. Barres, J. Martín \& N. Garcia-Jacas. 2011. Lessons from Plectocephalus (Compositae, Cardueae-Centaureinae): ITS disorientation in annuals and Beringian dispersal as revealed by molecular analyses. Annals of Botany 108: 263-277.

Takayama, K., P. López-Sepúlveda, G. Kohl, J. Novak \& T.F. Stuessy. 2013. Development of microsatellite markers in Robinsonia (Asteraceae) an endemic genus of the Juan Fernández Archipelago, Chile. Conservation Genetics Resources 5: 63-67.

Takayama, K., P. López-Sepúlveda, J. Greimler, D.J. Crawford, P. Peñailillo, M. Baeza, E. Ruiz, G. Kohl, K. Tremetsberger, A. Gatica, L. Letelier, P. Novoa, J. NovaK \& T.F. STUESSY. 2014. Relationships and genetic consequences of contrasting modes of speciation among endemic species of Robinsonia (Asteraceae, Senecioneae) of the Juan Fernández Archipelago, Chile, based on AFLPs and SSRs. New Phytologist 205: 415-428. 
TOKUOKA, T. 2007. Molecular phylogenetic analysis of Euphorbiaceae sensu stricto based on plastid and nuclear DNA sequences and ovule and seed character evolution. Journal of Plant Research 120: 511-522.

Toro-NúÑEz, O., I.A. Al-Shehbaz \& M.E. Mort. 2015. Phylogenetic study with nuclear and chloroplast data and ecological niche reveals Atacama (Brassicaceae), a new monotypic genus endemic from the Andes of the Atacama Desert, Chile. Plant Systematics and Evolution 301: 13771396.

TortosA, R.D. 1992. El complejo Retanilla-Talguenea-Trevoa (Rhamnaceae). Darwiniana 31: 223-252.

Trénel, P., M.H.G. Gustafsson, W.J. Baker, C.B. AsmussenLange, J. Dransfield, F. Borchsenius. 2007. Mid-Tertiary dispersal, not Gondwanan vicariance explains distribution patterns in the wax palm subfamily (Ceroxyloideae: Arecaceae). Molecular Phylogenetics and Evolution 45(1): 272-288.

Tu, T., S. Volis, M.O. Dillon, H. Sun \& J. Wen. 2010. Dispersals of Hyoscyameae and Mandragoreae (Solanaceae) from the New World to Eurasia in the early Miocene and their biogeographic diversification within Eurasia. Molecular Phylogenetics and Evolution 57: 1226-1237.

Unión Internacional para la Conservación de la Naturaleza (UICN). 2013. IUCN Red List of Threatened Species. Version 2013.2. URL: <www.iucnredlist.org>.

Ulibarri, E.A. 1996. Sinopsis de Caesalpinia y Hoffmannseggia (Leguminosae-Caesalpinioideae) de Sudamérica. Darwiniana 34(1-4): 299-348.

Ulibarri, E.A. 2008. Los géneros de Caesalpinioideae (Leguminosae) presentes en Sudamérica. Darwiniana 46(1): 69-163.

UlloA, C., J.L. ZARUCChI \& B. LeÓN. 2004. Diez años de adiciones a la flora del Perú: 1993-2003. Arnaldoa Edición Especial Nov. 2004: 1-242.

VAmosi, J.C. \& J.R.U. Wilson. 2008. Nonrandom extinction leads to elevated loss of angiosperm evolutionary history. Ecology Letters 11: 1047-1053.

Vargas, O. \& S. Madriñán. 2012. Preliminary Phylogeny of Diplostephium (Asteraceae): Speciation Rate and Character Evolution. Lundellia 15: 1-15.

Vidal-Russell, R. \& D.L. Nickrent. 2008. Evolutionary relationships in the showy mistletoe family (Loranthaceae). American Journal of Botany 95(8): 1015-1029.

Viruel, J., J.G. Segarra-Moragues, E. Pérez-Collazos, L. Villar \& P. CATALÁn. 2010. Systematic Revision of the Epipetrum Group of Dioscorea (Dioscoreaceae) Endemic to Chile. Systematic Botany 35(1): 40-63.

VolPONI, C.R. 1999. Caryophyllaceae. In: F.O. Zuloaga \& O. Morrone. Catálogo de las plantas vasculares de la República Argentina II. Monographs in Systematic Botany from the Missouri Botanical Garden, Vol. 74: 504-523.

Wallace, R. 1994. Appendix III. Phylogenetic analysis of Eriosyce. In: F. Kattermann (ed.), Eriosyce (Cactaceae). The genus revised and amplified. Succulent Plant Research 1: $125-130$.

Wallace, R.S. \& A.C. Gibson. 2002. Evolution and systematics. In: P.S. Nobel (ed.). Cacti. Biology and uses. University of California Press: 1-21.

WaLter, H. 2008. Floral biology phytogeography and systematics of Eriosyce subgenus Neoporteria (Cactaceae). Bradleya 26: 75-98.

Wang, W., A.M. Lu, Y. Ren, M.E. Endress \& Z.D. Chen. 2009. Phylogeny and classification of Ranunculales: Evidence from four molecular loci and morphological data. Perspectives in Plant Ecology, Evolution and Systematics 11: 81-110.

WArwick, S.I., C.A. SAuder \& I.A. Al-Shehbaz. 2011. Systematic position of Ivania, Scoliaxon, and Phravenia (Brassicaceae). Taxon 60(4): 1156-1164.

Weber, A. 2004. Gesneriaceae. In: K. Kubitzki (ed.). The Families and Genera of Vascular Plants, Vol. 7, pp. 63-158. Springer, New York.

Webster, G.L. 1994. Synopsis of the genera and suprageneric taxa of Euphorbiaceae. Annals of the Missouri Botanical Garden 81(1): 33-144.

Webster, G.L. 2014. Euphorbiaceae. In: K. Kubitzki (ed.). The Families and Genera of Vascular Plants, Vol. 11, pp. 51216. Springer, New York.

Weigend, M. 2004. Loasaceae. In: K. Kubitzki (ed.). The Families and Genera of Vascular Plants, Vol. 6, pp. 239-254. Springer, New York.

WeIGEND, M. 2005. Notes on the floral morphology in Vivianiaceae (Geraniales). Plant Systematics and Evolution 253: 125131.

Weigend, M. 2007. Ledocarpaceae. In: K. Kubitzki (ed.). The Families and Genera of Vascular Plants, Vol. 9, pp. 213220. Springer, New York.

Weigend, M., M. Gottschling, S. Hoot \& M. Ackermann. 2004. A preliminary phylogeny of Loasaceae subfam. Loasoideae (Angiospermae: Cornales) based on trnL (UAA) sequence data, with consequences for systematics and historical biogeography. Organisms, Diversity \& Evolution 4: 73-90.

WiLson, P.G. 2011. Myrtaceae. In: K. Kubitzki (ed.). The Families and Genera of Vascular Plants, Vol. 10, pp. 212-271. Springer, New York.

Wilson, C.A. \& C.L. CAlvin. 2006a. Character divergences and convergences in canopy-dwelling Loranthaceae. Botanical Journal of the Linnean Society 150: 101-113.

Wilson, C.A. \& C.L. CAlvin. 2006b. An origin of aerial branch parasitism in the mistletoe family, Loranthaceae. American Journal of Botany 93(5): 787-796.

Woo, V.L., M.M. FunKe, J.F. SMith, P.J. LockHART \& P.J. GARnockJones. 2011. New World origins of Southwest Pacific Gesneriaceae: Multiple movements across and within the South Pacific. International Journal of Plant Science 172(3): 434-457.

Wurdack, K.J., P. Hoffmann \& M.W. Chase. 2005. Molecular phylogenetic analysis of uniovulate Euphorbiaceae (Euphorbiaceae sensu stricto) using plastid rbcL and trnL-F DNA sequences. American Journal of Botany 92(8): 1397-1420.

Zizka, G., R. Horres, E.C. Nelson \& K. Weising. 1999. Revision of the genus Fascicularia Mez (Bromeliaceae). Botanical Journal of the Linnean Society 129: 315-332.

ZizKa, G., K. Trumpler \& O. ZöLlner. 2002. Revision of the genus Ochagavia (Bromeliaceae, Bromelioideae). Willdenowia 32: 331-350.

Zizka, G., M. Schmidt, K. Schulte, P. Novoa, R. Pinto \& K. KÖNIG. 2009. Chilean Bromeliaceae: diversity, distribution 
Consideraciones de las familias y géneros endémicos de Chile: Urbina-CASANOva, R. ET AL.

and evaluation of conservation status. Biodiversity Conservation 18: 2449-2471.

Zuloaga, F.O. \& O. Morrone. 1999. Catálogo de las plantas vasculares de la República Argentina II. Monographs in Systematic Botany from the Missouri Botanical Garden 74: 1-1331.

Zuloaga, F.O., O. Morrone \& D. Rodríguez. 1999. Análisis de la biodiversidad en plantas vasculares de la Argentina. Kurtziana 27: 17-167.

Zuloaga, F.O., O. Morrone \& M.J. Belgrano. 2008. Catálogo de las Plantas Vasculares del Cono Sur (Argentina, Sur de Brasil, Chile, Paraguay y Uruguay). Monographs in Systematic Botany from the Missouri Botanical Garden 107: 1-3348.

Recibido: 27.06 .14

Aceptado: 16.03 .15 